OPEN ACCESS

Edited by:

Marta Carrasco,

University of Lisbon, Portugal

Reviewed by:

Stevan Pecic,

California State University,

United States

Marcus Scotti,

Federal University of Paraiba, Brazil

*Correspondence:

Fernanda Andreia Rosa farosa@uem.br

Specialty section:

This article was submitted to Translational Pharmacology,

a section of the journal

Frontiers in Pharmacology

Received: 04 August 2020 Accepted: 15 September 2020

Published: 07 October 2020

Citation:

Camargo JdNA, Pianoski KE, dos Santos MG, Lazarin-Bidóia D, Volpato H, Moura S, Nakamura CV and Rosa FA (2020) Antiparasitic Behavior of Trifluoromethylated Pyrazole 2-Amino-1,3,

4-thiadiazole Hybrids and Their Analogues: Synthesis and Structure-Activity Relationship.

Front. Pharmacol. 11:591570. doi: 10.3389/fphar.2020.591570

\section{Antiparasitic Behavior of Trifluoromethylated Pyrazole 2- Amino-1,3,4-thiadiazole Hybrids and Their Analogues: Synthesis and Structure-Activity Relationship}

\author{
Jeniffer do Nascimento Ascencio Camargo ${ }^{1}$, Karlos Eduardo Pianoski ${ }^{1}$, \\ Mariellen Guilherme dos Santos ${ }^{1}$, Danielle Lazarin-Bidóia ${ }^{2}$, Hélito Volpato ${ }^{2}$, \\ Sidnei Moura ${ }^{3}$, Celso Vataru Nakamura ${ }^{2}$ and Fernanda Andreia Rosa ${ }^{1 *}$ \\ ${ }^{1}$ Departamento de Química, Universidade Estadual de Maringá (UEM), Maringá, Brazil, ${ }^{2}$ Laboratório de Inovação \\ Tecnológica no Desenvolvimento de Fármacos e Cosméticos, Departamento de Ciências Básicas da Saúde, Universidade \\ Estadual de Maringá (UEM), Maringá, Brazil, ${ }^{3}$ Instituto de Biotecnologia, Universidade de Caxias do Sul (UCS), Caxias do \\ Sul, Brazil
}

A series of trifluoromethylated pyrazole thiosemicarbazone, trifluromethylated pyrazole isothiosemicarbazone, and trifluoromethylated pyrazole 2-amino-1,3,4-thiadiazole hybrids were synthesized and evaluated in vitro against the promastigote form of Leishmania amazonensis and the epimastigote form of Trypanosoma cruzi, the pathogens causing the neglected tropical diseases leishmaniasis and Chagas disease, respectively. The results show the potential of these compounds regarding their antiparasitic properties. Studies on the structure-activity relationship demonstrated that compounds containing a bulky group at the para position of the phenyl ring attached to the 5-position of the pyrazole core had better antiparasitic effects. Among the substituents attached at the 3-position of the pyrazole ring, the insertion of the 2-amino-1,3,4thiadiazole nucleus led to the most potent compounds compared to the thiosemicarbazone derivative.

Keywords: trifluoromethylated pyrazoles, thiadiazole, thiosemicarbazone, antileishmanial, antitrypanosomal

\section{INTRODUCTION}

Every year, more than 700,000 deaths occur due to infectious diseases caused by parasites (Habercom, 2016). Among them, neglected tropical diseases (NTD) are responsible for about 530,000 deaths (WHO). Leishmaniasis is a NTD caused by Leishmania species and transmitted by the phlebotomine sandfly vector that can be found throughout the world, but mostly in developing countries, putting at risk more than 350 million lives (Habercom, 2016; Gradoni et al., 2017).

The drugs that are used nowadays for the treatment of leishmaniasis include pentavalent antimonials as first-line drugs, such as meglumine antimoniate. In case of resistance, other drugs are used like amphotericin B, paromomycin, miltefosine, and pentamidine (Alvar et al., 2012; 
Sangshetti et al., 2015; Hurrell et al., 2016). However, all these drugs present high toxicity and several collateral effects, beyond resistance of the protozoan to the drugs (Sangshetti et al., 2015).

Furthermore, Chagas disease (CD), also known as American trypanosomiasis, is another NTD that also occurs in developing countries, caused by the vector-borne flagellate protozoan parasite Trypanosoma cruzi. Chagas disease has infected over 20 million people in Central and South America and is responsible for around 20,000 deaths per year. Current treatments for Chagas disease are based on two old drugs, benznidazole (BZN) and nifurtimox (NFX) (Goupil and McKerrow, 2014; Chain et al., 2019). They are more effective in the acute phase of the disease, show several side effects, and require prolonged treatment. Therefore, there is remarkable urgency for the development of effective, inexpensive, and safe drugs for the treatment of NTD such as leishmaniasis and Chagas disease.

Several studies have shown the potential pharmacological effects of nitrogen-containing five-membered heterocycles. Pyrazole-based compounds have been reported as important small molecules in drug discovery for NTDs (Bekhit et al., 2015; Monteiro et al., 2019). For instance, Bekhit and coworkers in 2015 reported the antileishmanial activity of heterocycle hybrids containing the pyrazole moiety on Leishmania aethiopica (Figure 1A), whereas Monteiro and coworkers in 2019 reported the trypanocidal activity of pyrazole derivatives on T. cruzi (Figure 1B).

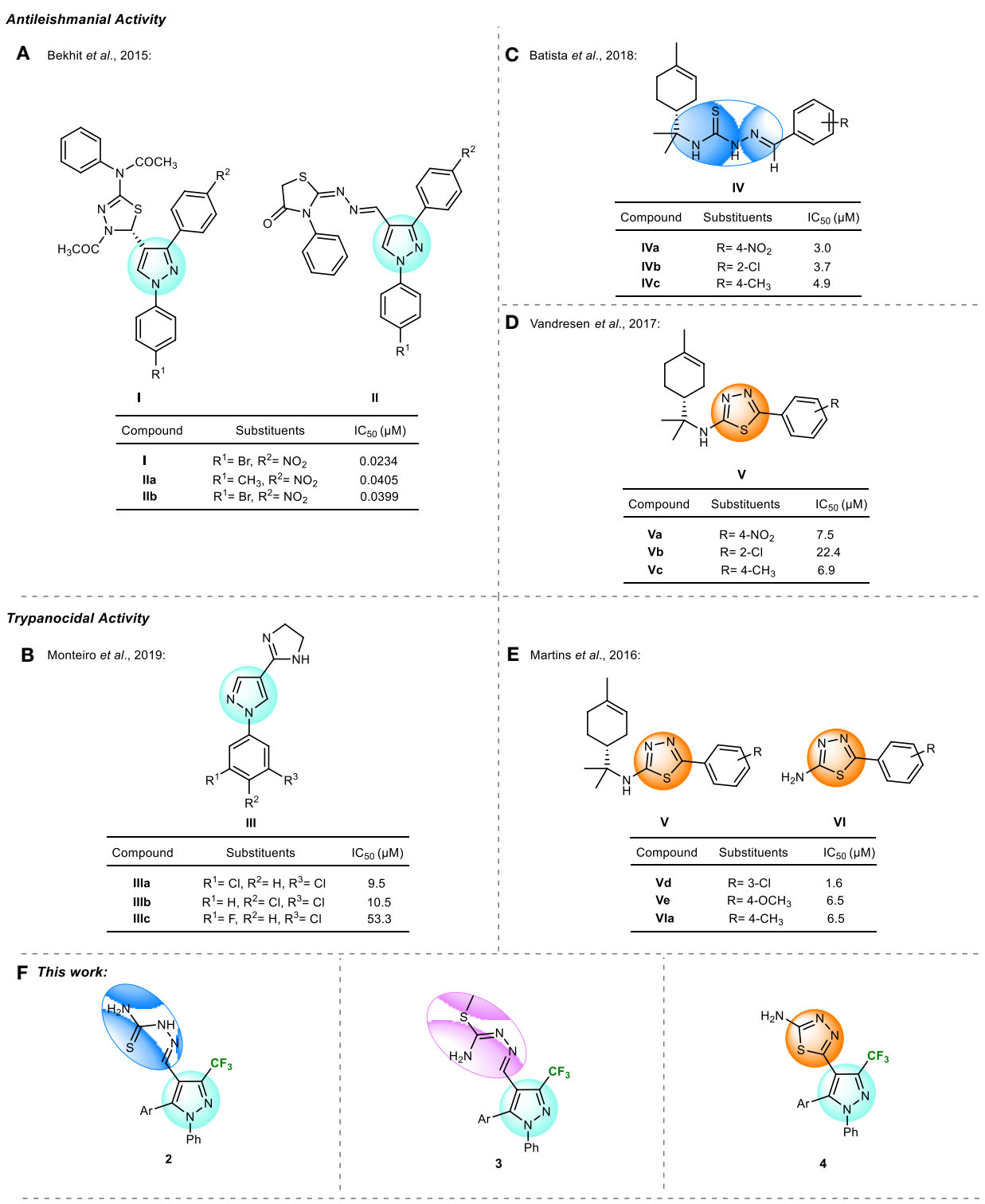

FIGURE 1 | (A-E) Previews works that related structures with antileishmanial and trypanocidal activity. (F) Structures proposed in this work. 
Moreover, 1,3,4-thiadiazole derivatives have exhibited a broad spectrum of pharmacological properties. They have a mesoionic characteristic that allow strong interactions with biomolecules. Also, the sulfur atom in this heterocycle imparts improved liposolubility. More specifically, 2-amino-1,3,4thiadiazole series (Jain et al., 2013; Martins et al., 2016; Vandresen et al., 2017; Serban, 2019; Freitas et al., 2020) and their thiosemicarbazone analogue (De Melos et al., 2015; Batista et al., 2019) have been studied for potential antileishmanial and trypanocidal activity (Figures 1C-E). Comparing the results reported for compounds IV and V, the 2-amino-1,3,4-thiadiazole derivatives were less active than the thiosemicarbazone derivatives (Figures 1C, D).

Additionally, the presence of fluorine atoms in organic compounds affect the chemical, biological, and physical properties of the molecules. Therefore, a trifluoromethyl group $\left(-\mathrm{CF}_{3}\right)$ can improve the pharmacological activities of compounds bearing this moiety (Petrov, 2009).

With the purpose of developing potent antiprotozoal compounds, that are less toxic and more selective, in this study, we adopted the molecular hybridization strategy for molecules design and synthesized a new series of trifluoromethylated pyrazoles and thiosemicarbazone/Smethylated thiosemicarbazone/2-amino 1,3,4-thiadiazoles hybrids (Figure 1F), and evaluated their antiprotozoal activity against the promastigote form of Leishmania amazonensis and the epimastigote form of Trypanosoma cruzi.

\section{EXPERIMENTAL SECTION}

\section{General Synthetic Procedure and Spectra Data}

Synthesis of 5-aryl-4-[(2-CarbamothioylHydrazinylidene)Methyl]-3-Trifluoromethyl-1-Phenyl$1 \mathrm{H}$-Pyrazole (2a-f)

\section{General Method}

The cyclocondensation reaction was performed according to the methodology described by Pianoski et al. (2020). The trifluoromethylated $\beta$-enamino diketone 1 (1a: $0.229 \mathrm{~g} ; \mathbf{1 b}$ : 0.317 g; 1c: 0.334 g; 1d: 0.378 g; 1e: 0.334 g; 1f: $0.329,1.0$ mmol, 1.0 equiv.) was solubilized in $\mathrm{MeCN}(10.0 \mathrm{ml})$, then added phenylhydrazine $(0.108 \mathrm{~g}, 1.0 \mathrm{mmol}, 1.0$ equiv) and boron trifluoride diethyl etherate solution $46.5 \%(0.400 \mathrm{ml}, 1.5 \mathrm{mmol}$, 1.5 equiv.). The mixture was stirred under reflux for $7 \mathrm{~h}$. In sequence, the reaction mixture was cooled to room temperature, added thiosemicarbazide $(0.276 \mathrm{~g}, 3.0 \mathrm{mmol}, 3.0$ equiv. $)$ and stirred for $30 \mathrm{~min}$. Then, the solvent was evaporated under vacuum and the residue was washed with a solution of $3 \%$ of $\mathrm{K}_{2} \mathrm{CO}_{3}(25 \mathrm{ml})$, extracted with dichloromethane $(3 \times 20 \mathrm{ml})$, and dried with anhydrous sodium sulfate. The solvent was evaporated under reduced pressure and the obtained residue was dissolved in hot ethyl ether $(5 \mathrm{ml})$ and cooled to $0^{\circ} \mathrm{C}$ which induced crystallization. The solid was filtered, washed with cold ethyl ether $(20 \mathrm{ml})$, and dried under vacuum.
4 - [(2-carbamothioyl-hydrazinylidene) methyl]-3 trifluoromethyl-5-(4-nitrophenyl)-1-phenyl-1H-pyrazole (2a): Yellow solid; $94 \%$ yield (0.408 g); mp $226.5{ }^{\circ} \mathrm{C} ;{ }^{1}$ H NMR (300.06 $\left.\mathrm{MHz}, \mathrm{DMSO}-d_{6}\right) \delta(\mathrm{ppm}) 6.49\left(s, 1 \mathrm{H}, \mathrm{CSNH}_{2}\right), 7.34-7.42(m$, $\left.5 \mathrm{H}, \mathrm{C}_{6} \mathrm{H}_{5}\right), 7.69\left(d, 2 \mathrm{H}, 4-\mathrm{NO}_{2}-\mathrm{C}_{6} \mathrm{H}_{4}, J=8.9 \mathrm{~Hz}\right), 8.01(s, 1 \mathrm{H}$, $\mathrm{CH}), 8.25\left(d, 2 \mathrm{H}, 4-\mathrm{NO}_{2}-\mathrm{C}_{6} \mathrm{H}_{4}, J=8.9 \mathrm{~Hz}\right), 8.31\left(s, 1 \mathrm{H}, \mathrm{CSNH}_{2}\right)$, $11.50(s, 1 \mathrm{H}, \mathrm{NH}) ;{ }^{13} \mathrm{C}$ NMR $\left(75.45 \mathrm{MHz}, \mathrm{DMSO}-d_{6}\right) \delta(\mathrm{ppm})$ $115.2\left(\mathrm{C}^{4}\right), 121.5\left(q, \underline{C F}_{3},{ }^{1} J_{\mathrm{C}-\mathrm{F}}=269.5 \mathrm{~Hz}\right), 123.8\left(4-\mathrm{NO}_{2}-\mathrm{C}_{6} \mathrm{H}_{4}\right)$, $126.0,129.5,129.5\left(\mathrm{C}_{6} \mathrm{H}_{5}\right), 132.3\left(4-\mathrm{NO}_{2}-\mathrm{C}_{6} \mathrm{H}_{4}\right), 132.6(\underline{\mathrm{CH}})$, $134.3\left(4-\mathrm{NO}_{2}-\mathrm{C}_{6} \mathrm{H}_{4}\right), 137.8\left(\mathrm{C}_{6} \mathrm{H}_{5}\right), 138.7\left(q, \mathrm{C}^{3},{ }^{2} J_{\mathrm{C}-\mathrm{F}}=37.4 \mathrm{~Hz}\right)$, $142.7\left(\mathrm{C}^{5}\right), 148.1\left(4-\mathrm{NO}_{2}-\mathrm{C}_{6} \mathrm{H}_{4}\right), 178.2(\mathrm{C}=\mathrm{S})$; HRMS (ESI+): calcd for $\mathrm{C}_{18} \mathrm{H}_{14} \mathrm{~F}_{3} \mathrm{~N}_{6} \mathrm{O}_{2} \mathrm{~S}^{+},[\mathrm{M}+\mathrm{H}]^{+}$: 435.0846, found 435.0864 .

4 - [ (2-carbamothioyl-hyd razinylidene) methyl] - 3 trifluoromethyl-5-(4-fluorophenyl)-1-phenyl-1 $H$-pyrazole (2b): White solid; $63 \%$ yield $(0.256 \mathrm{~g}) ; \mathrm{mp} 221.18^{\circ} \mathrm{C} ;{ }^{1} \mathbf{H}$ NMR $\left(500.13 \mathrm{MHz} \mathrm{CDCl}_{3}\right) \delta(\mathrm{ppm}) 6.23\left(\mathrm{~s}, 1 \mathrm{H}, \mathrm{CSNH}_{2}\right), 6.87(l \mathrm{~s}, 1 \mathrm{H}$, $\left.\mathrm{CSNH}_{2}\right), 7.09-7.15\left(m, 2 \mathrm{H}, 4-\mathrm{F}_{-} \mathrm{C}_{6} \mathrm{H}_{4}\right), 7.20-7.24$ ( $m, 4 \mathrm{H}, 4-\mathrm{F}-$ $\mathrm{C}_{6} \mathrm{H}_{4}$ and $\left.\mathrm{C}_{6} \mathrm{H}_{5}\right), 7.33-7.38\left(m, 3 \mathrm{H}, \mathrm{C}_{6} \mathrm{H}_{5}\right), 7.70(s, 1 \mathrm{H}, \mathrm{CH}), 9.61$ $(s, 1 \mathrm{H}, \mathrm{NH}) ;{ }^{13} \mathrm{C}$ NMR $\left(125.76 \mathrm{MHz}, \mathrm{CDCl}_{3}\right) \delta(\mathrm{ppm}) 114.1\left(\mathrm{C}^{4}\right)$, $116.5\left(d, 4-\mathrm{F}_{-} \mathrm{C}_{6} \mathrm{H}_{4},{ }^{2} J_{\mathrm{C}-\mathrm{F}}=22.0 \mathrm{~Hz}\right), 121.2\left(q, \underline{\mathrm{CF}}_{3},{ }^{1} J_{\mathrm{C}-\mathrm{F}}=\right.$ $269.9 \mathrm{~Hz}), 123.7\left(d, 4-\mathrm{F}_{-} \mathrm{C}_{6} \mathrm{H}_{4},{ }^{4} J_{\mathrm{C}-\mathrm{F}}=3.6 \mathrm{~Hz}\right), 125.4,129.0,129.4$ $\left(\mathrm{C}_{6} \mathrm{H}_{5}\right), 132.3\left(d, 4-\mathrm{F}_{-} \mathrm{C}_{6} \mathrm{H}_{4},{ }^{3} J_{\mathrm{C}-\mathrm{F}}=8.5 \mathrm{~Hz}\right), 133.7(\underline{\mathrm{CH}}), 138.2$ $\left(\mathrm{C}_{6} \mathrm{H}_{5}\right), 140.3\left(q, \mathrm{C}^{3},{ }^{2} J_{\mathrm{C}-\mathrm{F}}=38.2 \mathrm{~Hz}\right), 144.1\left(\mathrm{C}^{5}\right), 163.5(d, 4-\mathrm{F}-$ $\left.\mathrm{C}_{6} \mathrm{H}_{4},{ }^{1} J_{\mathrm{C}-\mathrm{F}}=252.1 \mathrm{~Hz}\right), 178.2(\mathrm{C}=\mathrm{S})$; HRMS (ESI+): calcd for $\mathrm{C}_{18} \mathrm{H}_{14} \mathrm{~F}_{4} \mathrm{~N}_{5} \mathrm{~S}^{+},[\mathrm{M}+\mathrm{H}]^{+}$: 408.0901, found 408.0918.

4-[(2-carbamothioyl-hydrazinylidene)methyl]-5-(4chlorophenyl)-3-trifluoromethyl-1-phenyl-1H-pyrazole (2c): White solid; $78 \%$ yield $(0.330 \mathrm{~g}) ; \mathrm{mp} 225.37^{\circ} \mathrm{C} ;{ }^{1} \mathbf{H}$ NMR $\left(500.13 \mathrm{MHz}, \mathrm{DMSO}-d_{6}\right) \delta(\mathrm{ppm}) 6.64\left(s, 1 \mathrm{H}, \mathrm{CSNH}_{2}\right), 7.32$ $7.44\left(m, 7 \mathrm{H}, 4-\mathrm{Cl}-\mathrm{C}_{6} \mathrm{H}_{4}\right.$ and $\left.\mathrm{C}_{6} \mathrm{H}_{5}\right), 7.52\left(d, 2 \mathrm{H}, 4-\mathrm{Cl}-\mathrm{C}_{6} \mathrm{H}_{4}, J=\right.$ $8.6 \mathrm{~Hz}), 7.97(s, 1 \mathrm{H}, \mathrm{CH}), 8.43\left(s, 1 \mathrm{H}, \mathrm{CSNH}_{2}\right), 11.51(s, 1 \mathrm{H}, \mathrm{NH})$; ${ }^{13} \mathrm{C}$ NMR (125.76 MHz, DMSO- $\left.d_{6}\right) \delta(\mathrm{ppm}) 114.6\left(\mathrm{C}^{4}\right), 121.4(q$, $\left.\underline{\mathrm{CF}}_{3},{ }^{1} J_{\mathrm{C}-\mathrm{F}}=269.2 \mathrm{~Hz}\right), 125.9\left(\mathrm{C}_{6} \mathrm{H}_{5}\right), 126.3,128.8\left(4-\mathrm{Cl}-\mathrm{C}_{6} \mathrm{H}_{4}\right)$, $129.2,129.3\left(\mathrm{C}_{6} \mathrm{H}_{5}\right), 132.4,134.8\left(4-\mathrm{Cl}_{-} \mathrm{C}_{6} \mathrm{H}_{4}\right), 133.0(\underline{\mathrm{CH}}), 137.9$ $\left(\mathrm{C}_{6} \mathrm{H}_{5}\right), 138.2\left(q, \mathrm{C}^{3},{ }^{2} J_{\mathrm{C}-\mathrm{F}}=37.3 \mathrm{~Hz}\right), 143.9\left(\mathrm{C}^{5}\right), 178.0(\mathrm{C}=\mathrm{S})$; HRMS (ESI+): calcd for $\mathrm{C}_{18} \mathrm{H}_{14} \mathrm{ClF}_{3} \mathrm{~N}_{5} \mathrm{~S}^{+},[\mathrm{M}+\mathrm{H}]^{+}$: 424.0605, found 424.0625 .

5-(4-bromophenyl)-4-[(2-carbamothioyl-hydrazinylidene) methyl]-3-trifluoromethyl-1-phenyl-1H-pyrazole (2d): White solid; $73 \%$ yield $(0.342 \mathrm{~g}) ; \mathrm{mp} 226.12{ }^{\circ} \mathrm{C} ;{ }^{1} \mathrm{H}$ NMR (500.13 $\left.\mathrm{MHz}, \mathrm{DMSO}-d_{6}\right) \delta(\mathrm{ppm}) 6.65\left(s, 1 \mathrm{H}, \mathrm{CSN}_{2}\right), 7.32-7-36(m$, $4 \mathrm{H}, \mathrm{C}_{6} \mathrm{H}_{5}$ and $\left.4-\mathrm{Br}_{-} \mathrm{C}_{6} \mathrm{H}_{4}\right), 7.42-7.45\left(m, 3 \mathrm{H}, \mathrm{C}_{6} \mathrm{H}_{5}\right), 7.65(d, 2 \mathrm{H}$, $\left.4-\mathrm{Br}-\mathrm{C}_{6} \mathrm{H}_{4}, J=8.5 \mathrm{~Hz}\right), 7.97(s, 1 \mathrm{H}, \mathrm{CH}), 8.43\left(s, 1 \mathrm{H}, \mathrm{CSNH}_{2}\right)$, $11.50(s, 1 \mathrm{H}, \mathrm{NH}) ;{ }^{13} \mathrm{C}$ NMR $\left(125.76 \mathrm{MHz}, \mathrm{DMSO}-d_{6}\right) \delta(\mathrm{ppm})$ $114.6\left(\mathrm{C}^{4}\right), 121.4\left(q, \mathrm{CF}_{3},{ }^{1} J_{\mathrm{C}-\mathrm{F}}=269.3 \mathrm{~Hz}\right), 125.8,126.6(4-\mathrm{Br}-$ $\left.\mathrm{C}_{6} \mathrm{H}_{4}\right), 129.2,129.3\left(\mathrm{C}_{6} \mathrm{H}_{5}\right), 129.3,131.7\left(4-\mathrm{Br}_{-} \mathrm{C}_{6} \mathrm{H}_{4}\right), 132.6$ $\left(\mathrm{C}_{6} \mathrm{H}_{5}\right), 131.9\left(4-\mathrm{Br}_{-} \mathrm{C}_{6} \mathrm{H}_{4}\right), 133.0(\underline{\mathrm{CH}}), 137.9\left(\mathrm{C}_{6} \mathrm{H}_{5}\right), 138.2(q$, $\left.\mathrm{C}^{3},{ }^{2} J_{\mathrm{C}-\mathrm{F}}=37.3 \mathrm{~Hz}\right), 143.9\left(\mathrm{C}^{5}\right), 178.0(\mathrm{C}=\mathrm{S})$; HRMS (ESI+): calcd for $\mathrm{C}_{18} \mathrm{H}_{14} \mathrm{BrF}_{3} \mathrm{~N}_{5} \mathrm{~S}^{+},[\mathrm{M}+\mathrm{H}]^{+}: 468.0100$, found 468.0102 .

4- [ (2-carba mothioyl-hyd razinylidene) methyl] - 3 trifluoromethyl-1,5-diphenyl- $\mathbf{H}$-pyrazole (2e): White solid; $70 \%$ yield $(0.272 \mathrm{~g}) ; \mathrm{mp} 211.27^{\circ} \mathrm{C} ;{ }^{\mathbf{1}} \mathrm{H}$ NMR $(500.13 \mathrm{MHz}$, DMSO- $\left.d_{6}\right) \delta(\mathrm{ppm}) 6.44\left(s, 1 \mathrm{H}, \mathrm{CSNH}_{2}\right), 6.84\left(s, 1 \mathrm{H}, \mathrm{CSNH}_{2}\right)$, 7.19-7.41 (m, 10H, $\mathrm{C}_{6} \mathrm{H}_{5} \mathrm{~A}$ and B), $7.79(s, 1 \mathrm{H}, \mathrm{CH}), 10.24(s, 1 \mathrm{H}$, $\mathrm{NH}) ;{ }^{13} \mathrm{C}$ NMR $\left(125.76 \mathrm{MHz}, \mathrm{DMSO}-d_{6}\right) \delta(\mathrm{ppm}) 114.0\left(\mathrm{C}^{4}\right)$, $121.3\left(q, \underline{C F}_{3},{ }^{1} J_{\mathrm{C}-\mathrm{F}}=270.0 \mathrm{~Hz}\right), 127.8,128.8,129.1,129.2,130.1$, 
130.2, $138.4\left(\mathrm{C}_{6} \mathrm{H}_{5}-\mathrm{A}\right.$ and $\left.\mathrm{B}\right), 133.9(\underline{\mathrm{CH}}), 140.3\left(q, \mathrm{C}^{3},{ }^{2} J_{\mathrm{C}-\mathrm{F}}=\right.$ $38.4 \mathrm{~Hz}), 145.1\left(\mathrm{C}^{5}\right), 178.0(\mathrm{C}=\mathrm{S})$; HRMS (ESI+): calcd for $\mathrm{C}_{18} \mathrm{H}_{15} \mathrm{~F}_{3} \mathrm{~N}_{5} \mathrm{~S}^{+},[\mathrm{M}+\mathrm{H}]^{+}$: 390.0995 , found 390.1002 .

4 - [ (2 - carba mothioyl-hydrazinylidene) methyl] -3 trifluoromethyl-5-(4-methoxyphenyl)-1-phenyl-1H-pyrazole (2f): White solid; $63 \%$ yield $(0.272 \mathrm{~g})$; $\mathrm{mp} 212.65^{\circ} \mathrm{C} ;{ }^{\mathbf{1}} \mathbf{H}$ NMR $\left(500.13 \mathrm{MHz}, \mathrm{CDCl}_{3}\right) \delta(\mathrm{ppm}) 3.83\left(\mathrm{~s}, 3 \mathrm{H}, 4-\mathrm{OCH}_{3}-\mathrm{C}_{6} \mathrm{H}_{4}\right), 6.39$ $\left(s, 1 \mathrm{H}, \mathrm{CSNH}_{2}\right), 6.91\left(d, 2 \mathrm{H}, 4-\mathrm{OCH}_{3}-\mathrm{C}_{6} \mathrm{H}_{4}, J=8.8 \mathrm{~Hz}\right), 6.95(s$, $\left.1 \mathrm{H}, \mathrm{CSNH}_{2}\right), 7.12\left(d, 2 \mathrm{H}, 4-\mathrm{OCH}_{3}-\mathrm{C}_{6} \mathrm{H}_{4}, J=8.8 \mathrm{~Hz}\right), 7.23-7.34$ $\left(m, 5 \mathrm{H}, \mathrm{C}_{6} \mathrm{H}_{5}\right), 7.76(s, 1 \mathrm{H}, \mathrm{C} \underline{\mathrm{H}}), 9.97(s, 1 \mathrm{H}, \mathrm{NH}) ;{ }^{13} \mathrm{C}$ NMR $\left(125.76 \mathrm{MHz}, \mathrm{CDCl}_{3}\right) \delta(\mathrm{ppm}) 55.5\left(4-\mathrm{OCH}_{3}-\mathrm{C}_{6} \mathrm{H}_{4}\right), 113.7\left(\mathrm{C}^{4}\right)$, 114.6, $119.6\left(4-\mathrm{OCH}_{3}-\mathrm{C}_{6} \mathrm{H}_{4}\right), 121.4\left(q, \underline{\mathrm{CF}}_{3},{ }^{1} J_{\mathrm{C}-\mathrm{F}}=269.7 \mathrm{~Hz}\right)$, 125.3, 128.7, $129.3\left(\mathrm{C}_{6} \mathrm{H}_{5}\right), 131.7\left(4-\mathrm{OCH}_{3}-\mathrm{C}_{6} \mathrm{H}_{4}\right), 134.0(\underline{\mathrm{CH}})$, $138.5\left(\mathrm{C}_{6} \mathrm{H}_{5}\right), 140.2\left(q, \mathrm{C}^{3},{ }^{2} J_{\mathrm{C}-\mathrm{F}}=38.2 \mathrm{~Hz}\right), 145.3\left(\mathrm{C}^{5}\right), 160.8(4-$ $\left.\mathrm{OCH}_{3}-\mathrm{C}_{6} \mathrm{H}_{4}\right), 178.0(\mathrm{C}=\mathrm{S})$; HRMS (ESI+): calcd for $\mathrm{C}_{19} \mathrm{H}_{17} \mathrm{~F}_{3} \mathrm{~N}_{5} \mathrm{OS}^{+},[\mathrm{M}+\mathrm{H}]^{+}$: 420.1100 , found 420.1121 .

\section{Synthesis of 5-Aryl-4-[(2-(S-Methyl- Carbonimidothioyl-Hydrazinylidene)Methyl]-3- Trifluoromethyl-1-Phenyl-1H-Pyrazole (3a-f) General Method}

In a solution of thiosemicarbazone derivatives 2 (2a: $0.434 \mathrm{~g} ; \mathbf{2 b}$ : 0.407 g; 2c: 0.423 g; 2 d: 0.468 g; 2e: 0.389 g; 2f: 0.419, 1.0 mmol, 1.0 equiv.) in DMSO (5.0 ml) was added sodium carbonate $(0.127 \mathrm{~g}, 1.2$ mmol, 1.2 equiv. $)$ and iodomethane solution $99 \%(0.063 \mathrm{ml}, 1.0$ mmol, 1.0 equiv.). The mixture was stirred under room temperature for $5 \mathrm{~min}$. Then, the product was washed with distilled water $(5 \times$ $15.0 \mathrm{ml}$ ) and filtered under vacuum. The obtained residue was dissolved in a mixture of hexane/ethyl acetate (4:1) and cooled to $0^{\circ}$ $\mathrm{C}$, which induced crystallization. The solid was filtered, washed with cold hexane $(20 \mathrm{ml})$ and dried under vacuum.

4-[(2-(S-methyl-carbonimidothioyl-hydrazinylidene) methyl]-3-trifluoromethyl-5-(4-nitrophenyl)-1-phenyl-1Hpyrazole (3a): Yellow solid; $85 \%$ yield $(0.367 \mathrm{~g})$; mp $190.7^{\circ} \mathrm{C} ;{ }^{1} \mathbf{H}$ NMR (300.06 MHz, $\left.\mathrm{CDCl}_{3}\right) \delta(\mathrm{ppm}) 2.45\left(\mathrm{~s}, 3 \mathrm{H}, \mathrm{SCH}_{3}\right), 5.22(s$, $\left.2 \mathrm{H}, \mathrm{NH}_{2}\right), 7.21-7.24\left(m, 2 \mathrm{H}, \mathrm{C}_{6} \mathrm{H}_{5}\right), 7.34-7.37\left(m, 3 \mathrm{H}, \mathrm{C}_{6} \mathrm{H}_{5}\right)$, $7.48\left(d, 2 \mathrm{H}, 4-\mathrm{NO}_{2}-\mathrm{C}_{6} \mathrm{H}_{4}, J=8.9 \mathrm{~Hz}\right), 8.21-8.24\left(m, 3 \mathrm{H}, 4-\mathrm{NO}_{2}-\right.$ $\mathrm{C}_{6} \mathrm{H}_{4}$ and $\mathrm{CH}$ ); ${ }^{13} \mathrm{C}$ NMR $\left(75.45 \mathrm{MHz}, \mathrm{CDCl}_{3}\right) \delta(\mathrm{ppm}) 12.8$ $\left(\mathrm{SCH}_{3}\right), 116.8\left(\mathrm{C}^{4}\right), 121.3\left(q, \underline{\mathrm{CF}}_{3},{ }^{1} J_{\mathrm{C}-\mathrm{F}}=269.8 \mathrm{~Hz}\right), 123.9(4-$ $\left.\mathrm{NO}_{2}-\mathrm{C}_{6} \mathrm{H}_{4}\right), 125.5,129.2,129.5\left(\mathrm{C}_{6} \mathrm{H}_{5}\right), 131.6,135.2\left(4-\mathrm{NO}_{2^{-}}\right.$ $\left.\mathrm{C}_{6} \mathrm{H}_{4}\right), 138.1\left(\mathrm{C}_{6} \mathrm{H}_{5}\right), 141.2\left(q, \mathrm{C}^{3},{ }^{2} J_{\mathrm{C}-\mathrm{F}}=38.1 \mathrm{~Hz}\right), 141.8\left(\mathrm{C}^{5}\right)$, $142.7(\underline{\mathrm{CH}}), 148.2\left(4-\mathrm{NO}_{2}-\mathrm{C}_{6} \mathrm{H}_{4}\right), 163.7$ (ㅌ-S); HRMS (ESI+): calcd for $\mathrm{C}_{19} \mathrm{H}_{16} \mathrm{~F}_{3} \mathrm{~N}_{6} \mathrm{O}_{2} \mathrm{~S}^{+},[\mathrm{M}+\mathrm{H}]^{+}:$449.1002, found 449.0994 .

4-[(2-( $S$-methyl-carbonimidothioyl-hydrazinylidene) methyl]-3-trifluoromethyl-5-(4-fluorophenyl)-1-phenyl-1Hpyrazole (3b): Beige solid; $78 \%$ yield $(0.316 \mathrm{~g})$; mp $145.5^{\circ} \mathrm{C} ;{ }^{1} \mathbf{H}$ NMR $\left(300.06 \mathrm{MHz}, \mathrm{CDCl}_{3}\right) \delta(\mathrm{ppm}) 2.45\left(\mathrm{~s}, 3 \mathrm{H}, \mathrm{SCH}_{3}\right), 5.28(s$, $\left.2 \mathrm{H}, \mathrm{NH}_{2}\right), 7.04-7.10\left(m, 2 \mathrm{H}, 4-\mathrm{F}-\mathrm{C}_{6} \mathrm{H}_{4}\right), 7.22-7.28$ ( $m, 5 \mathrm{H}, 4-\mathrm{F}-$ $\mathrm{C}_{6} \mathrm{H}_{4}$ and $\left.\mathrm{C}_{6} \mathrm{H}_{5}\right), 7.31-7.34\left(m, 3 \mathrm{H}, \mathrm{C}_{6} \mathrm{H}_{5}\right), 8.22(s, 1 \mathrm{H}, \mathrm{CH}) ;{ }^{13} \mathrm{C}$ NMR (75.45 MHz, $\left.\mathrm{CDCl}_{3}\right) \delta(\mathrm{ppm}) 12.8\left(\mathrm{SCH}_{3}\right), 116.2\left(\mathrm{C}^{4}\right)$, $116.2\left(d, 4-\mathrm{F}_{-} \mathrm{C}_{6} \mathrm{H}_{4},{ }^{2} J_{\mathrm{C}-\mathrm{F}}=22.0 \mathrm{~Hz}\right), 121.6\left(q, \mathrm{CF}_{3},{ }^{1} J_{\mathrm{C}-\mathrm{F}}=\right.$ $269.7 \mathrm{~Hz}), 124.7\left(d, 4-\mathrm{F}_{-} \mathrm{C}_{6} \mathrm{H}_{4},{ }^{4} J_{\mathrm{C}-\mathrm{F}}=3.6 \mathrm{~Hz}\right), 125.4,128.7,129.3$ $\left(\mathrm{C}_{6} \mathrm{H}_{5}\right), 132.5\left(d, 4-\mathrm{F}_{-} \mathrm{C}_{6} \mathrm{H}_{4},{ }^{3} J_{\mathrm{C}-\mathrm{F}}=8.5 \mathrm{~Hz}\right), 138.5\left(\mathrm{C}_{6} \mathrm{H}_{5}\right), 140.8$ $\left(q, \mathrm{C}^{3},{ }^{2} J_{\mathrm{C}-\mathrm{F}}=38.0 \mathrm{~Hz}\right), 143.5(\underline{\mathrm{CH}}), 143.7\left(\mathrm{C}^{5}\right), 163.2(\mathrm{C}-\mathrm{S}), 163.3$ $\left(d, 4-\mathrm{F}-\mathrm{C}_{6} \mathrm{H}_{4},{ }^{1} J_{\mathrm{C}-\mathrm{F}}=251.0 \mathrm{~Hz}\right)$; HRMS (ESI+): calcd for $\mathrm{C}_{19} \mathrm{H}_{16} \mathrm{~F}_{4} \mathrm{~N}_{5} \mathrm{~S}^{+},[\mathrm{M}+\mathrm{H}]^{+}$: 422.1057 , found 422.1053 .
5-(4-chlorophenyl)-4-[(2-(S-methyl-carbonimidothioylhydrazinylidene)methyl]-3-trifluoromethyl-1-phenyl-1Hpyrazole (3c): Yellow solid; $61 \%$ yield $(0.257 \mathrm{~g}) ; \mathrm{mp} 188.5{ }^{\circ} \mathrm{C} ;{ }^{1} \mathbf{H}$ NMR $\left(300.06 \mathrm{MHz}, \mathrm{CDCl}_{3}\right) \delta(\mathrm{ppm}) 2.45\left(s, 3 \mathrm{H}, \mathrm{SCH}_{3}\right), 5.22(s$, $\left.2 \mathrm{H}, \mathrm{NH}_{2}\right), 7.21-7.24\left(m, 2 \mathrm{H}, \mathrm{C}_{6} \mathrm{H}_{5}\right), 7.34-7.37\left(m, 3 \mathrm{H}, \mathrm{C}_{6} \mathrm{H}_{5}\right)$, $7.48\left(d, 2 \mathrm{H}, 4-\mathrm{Cl}-\mathrm{C}_{6} \mathrm{H}_{4}, J=8.9 \mathrm{~Hz}\right), 8.23\left(d, 2 \mathrm{H}, 4-\mathrm{Cl}_{-} \mathrm{C}_{6} \mathrm{H}_{4}, J=\right.$ $8.9 \mathrm{~Hz}), 8.25(s, 1 \mathrm{H}, \mathrm{CH}) ;{ }^{13} \mathrm{C}$ NMR $\left(75.45 \mathrm{MHz}, \mathrm{DMSO}-d_{6}\right) \delta$ (ppm) $12.8\left(\mathrm{~S}_{\underline{C}} H_{3}\right), 116.8\left(\mathrm{C}^{4}\right), 121.4\left(q, \underline{C F}_{3},{ }^{1} J_{\mathrm{C}-\mathrm{F}}=269.8 \mathrm{~Hz}\right)$, $125.9\left(\mathrm{C}_{6} \mathrm{H}_{5}\right), 126.3,128.8\left(4-\mathrm{Cl}-\mathrm{C}_{6} \mathrm{H}_{4}\right), 129.2,129.3\left(\mathrm{C}_{6} \mathrm{H}_{5}\right)$, 132.4, $134.8\left(4-\mathrm{Cl}-\mathrm{C}_{6} \mathrm{H}_{4}\right), 133.0(\underline{\mathrm{CH}}), 137.9\left(\mathrm{C}_{6} \mathrm{H}_{5}\right), 138.2\left(q, \mathrm{C}^{3}\right.$, $\left.{ }^{2} J_{\mathrm{C}-\mathrm{F}}=37.3 \mathrm{~Hz}\right), 143.9\left(\mathrm{C}^{5}\right), 178.0(\mathrm{C}-\mathrm{S})$; HRMS (ESI+): calcd for $\mathrm{C}_{19} \mathrm{H}_{16} \mathrm{ClF}_{3} \mathrm{~N}_{5} \mathrm{~S}^{+},[\mathrm{M}+\mathrm{H}]^{+}$: 438.0762 , found 438.0752 .

5-(4-Bromophenyl)-4-[(2-( $S$-methyl-carbonimidothioylhydrazinylidene) methyl]-3-trifluoromethyl-1-phenyl-1 Hpyrazole (3d): White solid; $80 \%$ yield $(0.373 \mathrm{~g}) ; \mathrm{mp} 137.4^{\circ} \mathrm{C} ;{ }^{\mathbf{1}} \mathbf{H}$ NMR $\left(300.06 \mathrm{MHz}, \mathrm{CDCl}_{3}\right) \delta(\mathrm{ppm}) 2.45\left(s, 3 \mathrm{H}, \mathrm{SCH}_{3}\right), 5.26(s$, $\left.2 \mathrm{H}, \mathrm{NH}_{2}\right), 7.14\left(d, 2 \mathrm{H}, 4-\mathrm{Br}-\mathrm{C}_{6} \mathrm{H}_{4}, J=8.6 \mathrm{~Hz}\right), 7.22-7.25(m, 2 \mathrm{H}$, $\left.\mathrm{C}_{6} \mathrm{H}_{5}\right), 7.32-7.36\left(m, 3 \mathrm{H}, \mathrm{C}_{6} \mathrm{H}_{5}\right), 7.51\left(d, 2 \mathrm{H}, 4-\mathrm{Br}_{-} \mathrm{C}_{6} \mathrm{H}_{4}, J=\right.$ $8.6 \mathrm{~Hz}), 8.22(s, 1 \mathrm{H}, \mathrm{CH}) ;{ }^{13} \mathrm{C}$ NMR $\left(75.45 \mathrm{MHz}, \mathrm{CDCl}_{3}\right) \delta$ (ppm) $\left.12.8\left(\mathrm{~S}_{\mathrm{CH}}\right)_{3}\right), 116.2\left(\mathrm{C}^{4}\right), 121.5\left(q, \underline{\mathrm{CF}}_{3},{ }^{1} J_{\mathrm{C}-\mathrm{F}}=269.6 \mathrm{~Hz}\right)$, 124.1, $127.6\left(4-\mathrm{Br}_{-} \mathrm{C}_{6} \mathrm{H}_{4}\right), 125.4,128.7,129.3\left(\mathrm{C}_{6} \mathrm{H}_{5}\right), 132.0,132.1$ $\left(4-\mathrm{Br}_{-} \mathrm{C}_{6} \mathrm{H}_{4}\right), 138.4\left(\mathrm{C}_{6} \mathrm{H}_{5}\right), 140.8\left(q, \mathrm{C}^{3},{ }^{2} J_{\mathrm{C}-\mathrm{F}}=37.9 \mathrm{~Hz}\right), 143.3$ ( $\mathrm{CH}), 143.4\left(\mathrm{C}^{5}\right), 163.3$ (C-S); HRMS (ESI+): calcd for $\mathrm{C}_{19} \mathrm{H}_{16} \mathrm{BrF}_{3} \mathrm{~N}_{5} \mathrm{~S}^{+},[\mathrm{M}+\mathrm{H}]^{+}$: 482.0256, found 482.0242 .

4-[(2-(S-methyl-carbonimidothioyl-hydrazinylidene) methyl]-3-trifluoromethyl-1,5-diphenyl-1H-pyrazole (3e): Beige solid; $73 \%$ yield $(0.283 \mathrm{~g}) ; \mathrm{mp} 165.65^{\circ} \mathrm{C} ;{ }^{1} \mathbf{H}$ NMR $\left(300.06 \mathrm{MHz}, \mathrm{CDCl}_{3}\right) \delta(\mathrm{ppm}) 2.44\left(s, 3 \mathrm{H}, \mathrm{SCH}_{3}\right), 5.25(s, 2 \mathrm{H}$, $\left.\mathrm{NH}_{2}\right), 7.23-7.39\left(m, 10 \mathrm{H}, \mathrm{C}_{6} \mathrm{H}_{5} \mathrm{~A}\right.$ and B), $8.24(\mathrm{~s}, 1 \mathrm{H}, \mathrm{CH}) ;{ }^{13} \mathrm{C}$ NMR $\left(75.45 \mathrm{MHz}, \mathrm{CDCl}_{3}\right) \delta(\mathrm{ppm}) 12.8\left(\mathrm{~S}_{\mathrm{CH}}\right), 116.0\left(\mathrm{C}^{4}\right)$, $121.6\left(q, \underline{C F}_{3},{ }^{1} J_{\mathrm{C}-\mathrm{F}}=269.6 \mathrm{~Hz}\right), 125.3,128.5,128.7,128.8,129.1$, $129.5,130.5,138.7\left(\mathrm{C}_{6} \mathrm{H}_{5}-\mathrm{A}\right.$ and $\left.\mathrm{B}\right), 140.6\left(q, \mathrm{C}^{3},{ }^{2} J_{\mathrm{C}-\mathrm{F}}=\right.$ $37.9 \mathrm{~Hz}), 143.7(\underline{\mathrm{CH}}), 144.8\left(\mathrm{C}^{5}\right), 163.1(\mathrm{C}-\mathrm{S})$; HRMS (ESI+): calcd for $\mathrm{C}_{19} \mathrm{H}_{17} \mathrm{~F}_{3} \mathrm{~N}_{5} \mathrm{~S}^{+},[\mathrm{M}+\mathrm{H}]^{+}:$404.1151, found 404.1140 .

4-[(2-(S-methyl-carbonimidothioyl-hydrazinylidene) methyl]-3-trifluoromethyl-5-(4-methoxyphenyl)-1-phenyl$1 \mathrm{H}$-pyrazole (3f): White solid; $68 \%$ yield $(0.284 \mathrm{~g}) ; \mathrm{mp} 126.2^{\circ} \mathrm{C}$; ${ }^{1} \mathbf{H}$ NMR $\left(300.06 \mathrm{MHz}, \mathrm{CDCl}_{3}\right) \delta(\mathrm{ppm}) 2.45\left(\mathrm{~s}, 3 \mathrm{H}, \mathrm{SCH}_{3}\right), 3.82$ $\left(s, 3 \mathrm{H}, 4-\mathrm{OCH}_{3}-\mathrm{C}_{6} \mathrm{H}_{4}\right), 5.31\left(s, 2 \mathrm{H}, \mathrm{NH}_{2}\right), 6.88\left(d, 2 \mathrm{H}, 4-\mathrm{OCH}_{3}\right.$ $\left.\mathrm{C}_{6} \mathrm{H}_{4}, J=8.2 \mathrm{~Hz}\right), 7.17\left(d, 2 \mathrm{H}, 4-\mathrm{OCH}_{3}-\mathrm{C}_{6} \mathrm{H}_{4}, J=8.8 \mathrm{~Hz}\right), 7.24-$ $7.33\left(m, 5 \mathrm{H}, \mathrm{C}_{6} \mathrm{H}_{5}\right), 8.22(s, 1 \mathrm{H}, \mathrm{CH}) ;{ }^{13} \mathrm{C}$ NMR $(75.45 \mathrm{MHz}$, $\left.\mathrm{CDCl}_{3}\right) \delta(\mathrm{ppm}) 12.8\left(\mathrm{SCH}_{3}\right), 55.4\left(4-\mathrm{OCH}_{3}-\mathrm{C}_{6} \mathrm{H}_{4}\right), 114.3$ $\left.\mathrm{OCH}_{3}-\mathrm{C}_{6} \mathrm{H}_{4}\right), 115.8\left(\mathrm{C}^{4}\right), 120.5\left(4-\mathrm{OCH}_{3}-\mathrm{C}_{6} \mathrm{H}_{4}\right), 121.6\left(q, \mathrm{CF}_{3}\right.$, $\left.{ }^{1} J_{\mathrm{C}-\mathrm{F}}=269.6 \mathrm{~Hz}\right), 125.4,128.4,129.1\left(\mathrm{C}_{6} \mathrm{H}_{5}\right), 131.8\left(4-\mathrm{OCH}_{3}-\right.$ $\left.\mathrm{C}_{6} \mathrm{H}_{4}\right), 138.8\left(\mathrm{C}_{6} \mathrm{H}_{5}\right), 140.4\left(q, \mathrm{C}^{3},{ }^{2} J_{\mathrm{C}-\mathrm{F}}=37.8 \mathrm{~Hz}\right), 144.0(\underline{\mathrm{CH}})$, $144.9\left(\mathrm{C}^{5}\right), 160.5\left(4-\mathrm{OCH}_{3}-\mathrm{C}_{6} \mathrm{H}_{4}\right), 162.9$ (C-S); HRMS (ESI+): calcd for $\mathrm{C}_{20} \mathrm{H}_{19} \mathrm{~F}_{3} \mathrm{~N}_{5} \mathrm{OS}^{+},[\mathrm{M}+\mathrm{H}]^{+}$: 434.1257, found 434.1254 .

\section{Synthesis of 4-[(2-Amino)-1,3,4-Thiadiazol-5-yl]-5- Aryl-3-Trifluoromethyl-1-Phenyl-1H-Pyrazole (4a-f) General Method}

In a solution of thiosemicarbazone derivatives 2 (2a: $0.434 \mathrm{~g}$; $\mathbf{2 b}$ : 0.407 g; 2c: 0.423 g; 2d: 0.468 g; 2e: 0.389 g; 2f: 0.419, 1.0 mmol, 1.0 equiv.) in dioxane $(5.0 \mathrm{ml})$ was added sodium carbonate ( $0.318 \mathrm{~g}, 3.0 \mathrm{mmol}, 3.0$ equiv.) and iodine ( $0.304 \mathrm{~g}, 1.2 \mathrm{mmol}, 1.2$ equiv.). The mixture was stirred under reflux for $4 \mathrm{~h}$. Then, the 
solvent was evaporated under vacuum and the residue was washed with a solution of $6 \%$ of $\mathrm{Na}_{2} \mathrm{~S}_{2} \mathrm{O}_{3}(25 \mathrm{ml})$, extracted with ethyl acetate $(3 \times 20 \mathrm{ml})$ and dried with anhydrous sodium sulfate. The obtained residue was dissolved in a mixture of hexane/ethyl acetate $(4: 1)$ and cooled to $0^{\circ} \mathrm{C}$, which induced crystallization. The solid was filtered, washed with cold hexane $(20 \mathrm{ml})$, and dried under vacuum.

4-[(2-amino)-1,3,4-thiadiazo-5-yl]-3-trifluoromethyl-5-(4nitrophenyl)-1-phenyl- $1 \mathrm{H}$-pyrazole (4a): White solid; $56 \%$ yield (0.242 g); mp $236.7^{\circ} \mathrm{C} ;{ }^{1} \mathbf{H}$ NMR (500.13 MHz, DMSO$\left.d_{6}\right) \delta(\mathrm{ppm}) 7.34\left(s, 2 \mathrm{H}, \mathrm{NH}_{2}\right), 7.42-7.45\left(m, 5 \mathrm{H}, \mathrm{C}_{6} \mathrm{H}_{5}\right), 7.67(d$, $\left.2 \mathrm{H}, 4-\mathrm{NO}_{2}-\mathrm{C}_{6} \mathrm{H}_{4}, J=8.8 \mathrm{~Hz}\right), 8.24\left(d, 2 \mathrm{H}, 4-\mathrm{NO}_{2}-\mathrm{C}_{6} \mathrm{H}_{4}, J=\right.$ $8.8 \mathrm{~Hz}) ;{ }^{13} \mathrm{C}$ NMR $\left(125.76 \mathrm{MHz}, \mathrm{DMSO}-d_{6}\right) \delta(\mathrm{ppm}) 111.7\left(\mathrm{C}^{4}\right)$, $121.0\left(q, \underline{C F}_{3},{ }^{1} J_{\mathrm{C}-\mathrm{F}}=269.7 \mathrm{~Hz}\right), 123.6\left(4-\mathrm{NO}_{2}-\mathrm{C}_{6} \mathrm{H}_{4}\right), 126.1$, 129.3, $129.4\left(\mathrm{C}_{6} \mathrm{H}_{5}\right), 132.4,133.8\left(4-\mathrm{NO}_{2}-\mathrm{C}_{6} \mathrm{H}_{4}\right), 137.9\left(\mathrm{C}_{6} \mathrm{H}_{5}\right)$, $139.1\left(q, \mathrm{C}^{3},{ }^{2} J_{\mathrm{C}-\mathrm{F}}=37.0 \mathrm{~Hz}\right), 142.5\left(\mathrm{C}^{5}\right), 143.9(\mathrm{C}=\mathrm{N}), 148.2(4-$ $\mathrm{NO}_{2}-\mathrm{C}_{6} \mathrm{H}_{4}$ ), 169.8 ( $\underline{\mathrm{C}}-\mathrm{NH}_{2}$ ); HRMS (ESI+): calcd for $\mathrm{C}_{18} \mathrm{H}_{12} \mathrm{~F}_{3} \mathrm{~N}_{6} \mathrm{O}_{2} \mathrm{~S}^{+},[\mathrm{M}+\mathrm{H}]^{+}$: 433.0689 , found 433.0704 .

4-[(2-amino)-1,3,4-thiadiazo-5-yl]-3-trifluoromethyl-5-(4fluorophenyl)-1-phenyl-1H-pyrazole (4b): White solid; 53\% yield (0.245 g); mp $243.3^{\circ} \mathrm{C} ;{ }^{1} \mathbf{H}$ NMR (300.06 MHz, DMSO$\left.d_{6}\right) \delta(\mathrm{ppm}) 7.23-7.29\left(m, 2 \mathrm{H}, 4-\mathrm{F}-\mathrm{C}_{6} \mathrm{H}_{4}\right), 7.36-7.46(m, 9 \mathrm{H}, 4-\mathrm{F}-$ $\mathrm{C}_{6} \mathrm{H}_{4}, \mathrm{C}_{6} \mathrm{H}_{5}$ and $\left.\mathrm{NH}_{2}\right) ;{ }^{13} \mathrm{C}$ NMR $\left(75.45 \mathrm{MHz}, \mathrm{DMSO}-d_{6}\right) \delta$ (ppm) $112.2\left(\mathrm{C}^{4}\right), 116.6\left(d, 4-\mathrm{F}_{-} \mathrm{C}_{6} \mathrm{H}_{4},{ }^{2} J_{\mathrm{C}-\mathrm{F}}=21.9 \mathrm{~Hz}\right), 121.7(q$, $\left.\mathrm{CF}_{3},{ }^{1} J_{\mathrm{C}-\mathrm{F}}=269.1 \mathrm{~Hz}\right), 124.3\left(d, 4-\mathrm{F}_{-} \mathrm{C}_{6} \mathrm{H}_{4},{ }^{4} J_{\mathrm{C}-\mathrm{F}}=3.1 \mathrm{~Hz}\right), 126.6$, 129.8, $129.8\left(\mathrm{C}_{6} \mathrm{H}_{5}\right), 133.9\left(d, 4-\mathrm{F}_{-} \mathrm{C}_{6} \mathrm{H}_{4},{ }^{3} \mathrm{~J}_{\mathrm{C}-\mathrm{F}}=8.5 \mathrm{~Hz}\right), 138.7$ $\left(\mathrm{C}_{6} \mathrm{H}_{5}\right), 137.4\left(q, \mathrm{C}^{3},{ }^{2} J_{\mathrm{C}-\mathrm{F}}=38.4 \mathrm{~Hz}\right), 144.3\left(\mathrm{C}^{5}\right), 163.6(d, 4-\mathrm{F}-$ $\mathrm{C}_{6} \mathrm{H}_{4},{ }^{1} J_{\mathrm{C}-\mathrm{F}}=248.2 \mathrm{~Hz}$ ); HRMS (ESI+): calcd for $\mathrm{C}_{18} \mathrm{H}_{12} \mathrm{~F}_{4} \mathrm{~N}_{5} \mathrm{~S}^{+}$, $[\mathrm{M}+\mathrm{H}]^{+}:$406.0744, found 406.0736.

4-[(2-amino)-1,3,4-thiadiazo-5-yl]-5-(4-chlorophenyl)-3trifluoromethyl-1-phenyl-1H-pyrazole (4c): White solid; $54 \%$ yield (0.229 g); mp 224. ${ }^{\circ} \mathrm{C}$; ${ }^{1} \mathrm{H}$ NMR (300.06 MHz, DMSO- $\left.d_{6}\right) \delta$ (ppm) $7.33\left(s, 2 \mathrm{H}, \mathrm{NH}_{2}\right), 7.39-7.44\left(m, 7 \mathrm{H}, \mathrm{C}_{6} \mathrm{H}_{5}\right.$ and $4-\mathrm{Cl}-$ $\left.\mathrm{C}_{6} \mathrm{H}_{4}\right), 7.49\left(d, 2 \mathrm{H}, 4-\mathrm{Cl}-\mathrm{C}_{6} \mathrm{H}_{4}, J=8.5 \mathrm{~Hz}\right) ;{ }^{13} \mathrm{C}$ NMR $(75.45$ $\left.\mathrm{MHz}, \mathrm{DMSO}-d_{6}\right) \delta(\mathrm{ppm}) 111.4\left(\mathrm{C}^{4}\right), 121.1\left(q, \underline{\mathrm{CF}}_{3},{ }^{1} J_{\mathrm{C}-\mathrm{F}}=\right.$ $269.6 \mathrm{~Hz}), 126.0\left(\mathrm{C}_{6} \mathrm{H}_{5}\right), 126.1\left(4-\mathrm{Cl}-\mathrm{C}_{6} \mathrm{H}_{4}\right), 128.9,129.2\left(\mathrm{C}_{6} \mathrm{H}_{5}\right)$, 129.2, 132.6, $135.1\left(4-\mathrm{Cl}-\mathrm{C}_{6} \mathrm{H}_{4}\right), 138.0\left(\mathrm{C}_{6} \mathrm{H}_{5}\right), 138.7\left(q, \mathrm{C}^{3}\right.$, $\left.{ }^{2} J_{\mathrm{C}-\mathrm{F}}=36.8 \mathrm{~Hz}\right), 143.3\left(\mathrm{C}^{5}\right), 169.6\left(\underline{\mathrm{C}}-\mathrm{NH}_{2}\right)$; HRMS (ESI+): calcd for $\mathrm{C}_{18} \mathrm{H}_{12} \mathrm{ClF}_{3} \mathrm{~N}_{5} \mathrm{~S}^{+},[\mathrm{M}+\mathrm{H}]^{+}$: 422.0449, found 422.0471 .

4-[(2-amino)-1,3,4-thiadiazo-5-yl]-5-(4-Bromophenyl)-3trifluoromethyl-1-phenyl-1H-pyrazole (4d): White solid; $54 \%$ yield $(0.251 \mathrm{~g}) ; \mathrm{mp} 239.2^{\circ} \mathrm{C} ;{ }^{1} \mathbf{H}$ NMR $\left(300.06 \mathrm{MHz}, \mathrm{DMSO}-d_{6}\right) \delta$ (ppm) 7.31-7.33 (m, 4H, 4- $\mathrm{Br}_{-} \mathrm{C}_{6} \mathrm{H}_{4}$ and $\left.\mathrm{NH}_{2}\right), 7.37-7.45(m$, $\left.5 \mathrm{H}, \mathrm{C}_{6} \mathrm{H}_{5}\right), 7.62\left(d, 2 \mathrm{H}, 4-\mathrm{Br}_{-} \mathrm{C}_{6} \mathrm{H}_{4}, J=8.4 \mathrm{~Hz}\right) ;{ }^{13} \mathrm{C}$ NMR $(75.45$ $\left.\mathrm{MHz}, \mathrm{DMSO}-d_{6}\right) \delta(\mathrm{ppm}) 111.4\left(\mathrm{C}^{4}\right), 121.1\left(q, \underline{\mathrm{CF}}_{3},{ }^{1} J_{\mathrm{C}-\mathrm{F}}=\right.$ $269.7 \mathrm{~Hz}), 124.0,126.4\left(4-\mathrm{Br}-\mathrm{C}_{6} \mathrm{H}_{4}\right), 126.0,126.4,129.2\left(\mathrm{C}_{6} \mathrm{H}_{5}\right)$, 131.8, $132.8\left(4-\mathrm{Br}_{-} \mathrm{C}_{6} \mathrm{H}_{4}\right), 138.0\left(\mathrm{C}_{6} \mathrm{H}_{5}\right), 138.8\left(q, \mathrm{C}^{3},{ }^{2} J_{\mathrm{C}-\mathrm{F}}=\right.$ $37.0 \mathrm{~Hz}), 143.3\left(\mathrm{C}^{5}\right)$; HRMS (ESI+): calcd for $\mathrm{C}_{18} \mathrm{H}_{12} \mathrm{BrF}_{3} \mathrm{~N}_{5} \mathrm{~S}^{+}$, $[\mathrm{M}+\mathrm{H}]^{+}:$465.9943, found 465.9974 .

4-[(2-amino)-1,3,4-thiadiazo-5-yl]-3-trifluoromethyl-1,5diphenyl- $1 \mathrm{H}$-pyrazole (4e): White solid; $52 \%$ yield $(0.201 \mathrm{~g}) ; \mathrm{mp}$ 275.5; ${ }^{1} \mathrm{H}$ NMR (300.06 MHz, DMSO- $\left.d_{6}\right) \delta(\mathrm{ppm}) 7.27(s, 2 \mathrm{H}$, $\left.\mathrm{NH}_{2}\right), 7.34-7.44\left(m, 10 \mathrm{H}, \mathrm{C}_{6} \mathrm{H}_{5} \mathrm{~A}\right.$ and B); ${ }^{13} \mathrm{C}$ NMR $(75.45 \mathrm{MHz}$, DMSO- $\left.d_{6}\right) \delta(\mathrm{ppm}) 111.3\left(\mathrm{C}^{4}\right), 121.1\left(q, \underline{\mathrm{CF}}_{3},{ }^{1} J_{\mathrm{C}-\mathrm{F}}=269.8 \mathrm{~Hz}\right)$, $125.9,127.1,128.7,129.1,129.1,130.1,130.7,138.2\left(\mathrm{C}_{6} \mathrm{H}_{5}-\mathrm{A}\right.$ and B), 138.7 ( $\left.q, \mathrm{C}^{3},{ }^{2} J_{\mathrm{C}-\mathrm{F}}=36.9 \mathrm{~Hz}\right), 144.5\left(\mathrm{C}^{5}\right)$; HRMS (ESI+): calcd for $\mathrm{C}_{18} \mathrm{H}_{13} \mathrm{~F}_{3} \mathrm{~N}_{5} \mathrm{~S}^{+},[\mathrm{M}+\mathrm{H}]^{+}: 388.0838$, found 388.0860 .

4-[(2-amino)-1,3,4-thiadiazo-5-yl]-3-trifluoromethyl-5-(4methoxyphenyl)-1-phenyl-1H-pyrazole (4f): White solid; $50 \%$ yield (0.208 g); mp $239.1^{\circ} \mathrm{C}$; ${ }^{1} \mathrm{H}$ NMR (300.06 MHz, DMSO- $\left.d_{6}\right) \delta$ (ppm) $2.45\left(s, 3 \mathrm{H}, \mathrm{SCH}_{3}\right), 3.75\left(s, 3 \mathrm{H}, 4-\mathrm{OCH}_{3}-\mathrm{C}_{6} \mathrm{H}_{4}\right), 6.95(d$, $\left.2 \mathrm{H}, 4-\mathrm{OCH}_{3}-\mathrm{C}_{6} \mathrm{H}_{4}, J=8.8 \mathrm{~Hz}\right), 7.25-7.28\left(m, 4 \mathrm{H}, 4-\mathrm{OCH}_{3}-\mathrm{C}_{6} \mathrm{H}_{4}\right.$ and $\left.\mathrm{NH}_{2}\right), 7.35-7.43\left(m, 5 \mathrm{H}, \mathrm{C}_{6} \mathrm{H}_{5}\right) ;{ }^{13} \mathrm{C}$ NMR $(75.45 \mathrm{MHz}$, DMSO- $\left.d_{6}\right) \delta(\mathrm{ppm}) 55.2\left(4-\mathrm{OCH}_{3}-\mathrm{C}_{6} \mathrm{H}_{4}\right), 111.2\left(\mathrm{C}^{4}\right), 114.3(4-$ $\left.\mathrm{OCH}_{3}-\mathrm{C}_{6} \mathrm{H}_{4}\right), 119.0\left(4-\mathrm{OCH}_{3}-\mathrm{C}_{6} \mathrm{H}_{4}\right), 121.2\left(q, \mathrm{CF}_{3},{ }^{1} J_{\mathrm{C}-\mathrm{F}}=\right.$ $269.6 \mathrm{~Hz}), 125.9,129.0,129.2\left(\mathrm{C}_{6} \mathrm{H}_{5}\right), 132.2\left(4-\mathrm{OCH}_{3}-\mathrm{C}_{6} \mathrm{H}_{4}\right)$, $138.4\left(\mathrm{C}_{6} \mathrm{H}_{5}\right), 139.1\left(q, \mathrm{C}^{3},{ }^{2} J_{\mathrm{C}-\mathrm{F}}=36.5 \mathrm{~Hz}\right), 144.5\left(\mathrm{C}^{5}\right), 160.4(4-$ $\mathrm{OCH}_{3}-\mathrm{C}_{6} \mathrm{H}_{4}$ ); HRMS (ESI+): calcd for $\mathrm{C}_{19} \mathrm{H}_{15} \mathrm{~F}_{3} \mathrm{~N}_{5} \mathrm{OS}^{+}$, [M $+\mathrm{H}]^{+}$: 418.0944, found 418.0934.

\section{Synthesis of 5-Aryl-3-Trifluoromethyl-4-Formyl-1- Phenyl-1H-Pyrazole (5a) \\ General Method}

The TBED 1a $(0.344 \mathbf{g}, 1.0 \mathrm{mmol}, 1.0$ equiv. $)$ was solubilized in $\mathrm{MeCN}(8 \mathrm{ml})$, then added phenylhydrazine $(0.108 \mathrm{~g}, 1.0 \mathrm{mmol}$, 1.0 equiv.) and boron trifluoride diethyl etherate solution $46.5 \%$ ( $0.400 \mathrm{ml}, 1.5 \mathrm{mmol}, 1.5$ equiv.). The mixture was stirred under reflux for $7 \mathrm{~h}$. Next, reaction mixture was cooled to room temperature and the solvent was evaporated under vacuum. Then, the residue was washed with distilled water $(25 \mathrm{ml})$ extracted with dichloromethane $(3 \times 20 \mathrm{ml})$ and dried with anhydrous sodium sulfate. The solvent was evaporated under reduced pressure and the product was isolated on a silica gel chromatography column using a 95:5 mixture of hexane: ethyl acetate as the eluent.

3-trifluoromethyl-4-formyl-5-(4-nitrophenyl)-1-phenyl$\mathbf{1 H}$-pyrazole (5a): Orange solid; $84 \%$ yield $(0.311 \mathrm{~g}) ; \mathrm{mp} 155.2-$ $156.1^{\circ} \mathrm{C} ;{ }^{1} \mathbf{H}$ NMR $\left(300.06 \mathrm{MHz}, \mathrm{CDCl}_{3}\right) \delta(\mathrm{ppm}) 7.21-7.23(\mathrm{~m}$, $\left.2 \mathrm{H}, \mathrm{C}_{6} \mathrm{H}_{5}\right) 7.38-7.44\left(m, 3 \mathrm{H}, \mathrm{C}_{6} \mathrm{H}_{5}\right), 7.51\left(d, 2 \mathrm{H}, 4-\mathrm{NO}_{2}-\mathrm{C}_{6} \mathrm{H}_{4}\right.$, $J=8.8 \mathrm{~Hz}), 8.25\left(d, 2 \mathrm{H}, 4-\mathrm{NO}_{2}-\mathrm{C}_{6} \mathrm{H}_{4}, J=8.8 \mathrm{~Hz}\right), 10.03(s, 1 \mathrm{H}$, $\mathrm{CHO}) ;{ }^{13} \mathrm{C}$ NMR (75.45 MHz, $\left.\mathrm{CDCl}_{3}\right) \delta(\mathrm{ppm}) 119.0\left(\mathrm{C}^{4}\right), 120.6$ $\left(q, \underline{C F}_{3},{ }^{1} J_{\mathrm{C}-\mathrm{F}}=270.6 \mathrm{~Hz}\right), 123.9\left(4-\mathrm{NO}_{2}-\mathrm{C}_{6} \mathrm{H}_{4}\right), 125.6,129.7$, 129.9, $\left(\mathrm{C}_{6} \mathrm{H}_{5}\right), 131.8,133.2\left(4-\mathrm{NO}_{2}-\mathrm{C}_{6} \mathrm{H}_{4}\right), 137.4\left(\mathrm{C}_{6} \mathrm{H}_{5}\right), 143.7$ $\left(q, \mathrm{C}^{3},{ }^{2} J_{\mathrm{C}-\mathrm{F}}=39.4 \mathrm{~Hz}\right), 145.2\left(\mathrm{C}^{5}\right), 148.8\left(4-\mathrm{NO}_{2}-\mathrm{C}_{6} \mathrm{H}_{4}\right), 182.7$ ( $\mathrm{CHO}$ ); HRMS (ESI+): calcd for $\mathrm{C}_{17} \mathrm{H}_{11} \mathrm{~F}_{3} \mathrm{~N}_{3} \mathrm{O}_{3}{ }^{+},[\mathrm{M}+\mathrm{H}]^{+}$: 362.0747, found 362.0755 .

\section{RESULTS AND DISCUSSION}

A one-pot synthesis of thiosemicarbazone derivatives $2 \mathbf{a}-\mathbf{f}$ starting from trifluoromethylated $\beta$-enamino diketone (TBED) $\mathbf{1 a}-\mathbf{f}$ was developed based on a methodology previously reported by our research group (Pianoski et al., 2020) (Figure 2). This reaction exhibited high regioselectivity, good scope, and efficiency, providing six new thiosemicarbazone derivatives (2a-f) in moderate to excellent yields (63-94\%). The electronic effects of aryl group in the TBED displayed an important role, 


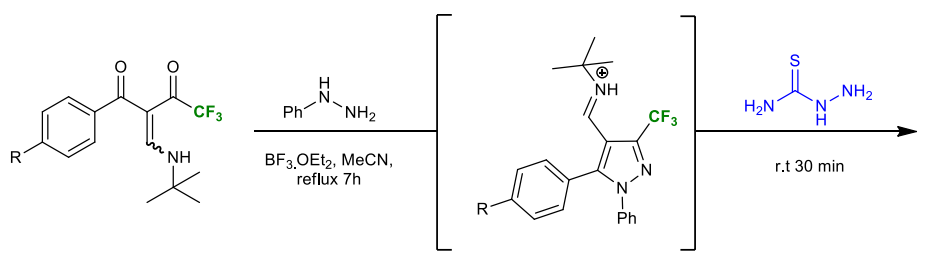

1

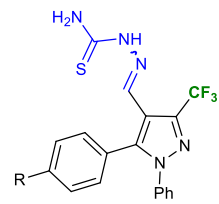

2
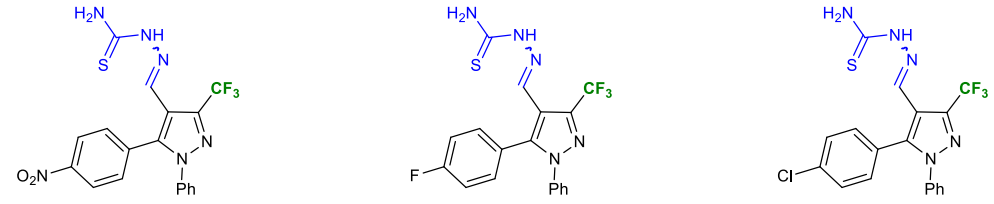

2b $(63 \%)$
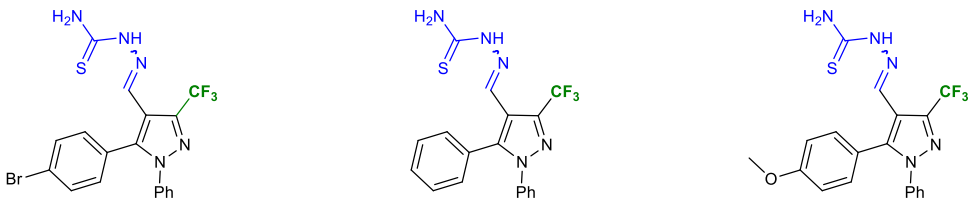

2d $(73 \%)$

2e $(70 \%)$

2f $(65 \%)$

FIGURE 2 | Substrate scope of thiosemicarbazones 2a-f. Reaction conditions: TBED 1a-f (1.0 mmol), phenylhydrazine (1.0 mmol), BF ${ }_{3} \mathrm{OEt} \mathrm{t}_{2}$ (1.5 mmol), MeCN $(10.0 \mathrm{ml})$, thiosemicarbazide $(3.0 \mathrm{mmol})$. Isolated yield after recrystallization in ethyl ether.

since aryl groups with electron withdrawing group had the best yields, while neutral and electron donors had the worst.

Next, we synthesized a series of $S$-methylated thiosemi carbazone derivatives $\mathbf{3 a - f}$ from the methylation reaction of the sulfur atom of their respective thiosemicarbazones $\mathbf{2 a - f}$. For the synthesis of these derivatives, we performed some tests where the optimal condition was found when the reaction was carried out in DMSO, at room temperature, for $5 \mathrm{~min}$, using 3.0 equiv. of iodomethane and 1.2 equivalents of $\mathrm{Na}_{2} \mathrm{CO}_{3}$. Satisfactorily, the methodology was simple and effective, and the products $3 \mathbf{a}-\mathbf{f}$ were obtained with moderate to good yields (61-85\%) (Figure 3).

The syntheses of the 1,3,4-thiadiazole-pyrazole hybrids $4 \mathrm{a}-\mathrm{f}$ were conducted from the oxidative cyclisation of their respective thiosemicarbazones 2a-f. Various methods have been reported for the synthesis of this core on literature (Hu et al., 2014; Haider et al., 2015), so we used two different oxidant agents and conditions for the cyclisation (Table 1). Initially, the compound $2 \mathrm{a}$ was subject to the oxidative cyclisation reaction using 1.2 equiv. of molecular iodine, 3.0 equivalents of $\mathrm{Na}_{2} \mathrm{CO}_{3}$, in THF, at room temperature. However, most of the starting material was recovered, even after prolonged reaction time (Entry 1, Table 1). On the other hand, when the reaction was carried out at reflux, the desired product 4 a was obtained with a yield of $44 \%$ (Entry 2, Table 1). The yield of the product 4 a was slightly improved when employed 1,4-dioxane reflux (Entry 3, Table 1). We also test ferric chloride as an oxidant. Nevertheless, a longer time was necessary for the total conversion to the desired product (Entries 4-6, Table 1). Thus, the best condition found for the oxidative cyclisation was using molecular iodine as oxidant with 3.0 equivalents of $\mathrm{Na}_{2} \mathrm{CO}_{3}$ under 1,4-dioxane reflux for $4 \mathbf{h}$ (Entry 3, Table $\mathbf{1}$ ).

The efficacy of the methodology was demonstrated when applied for the other thiosemicarbazones $2 \mathrm{~b}-\mathrm{f}$ containing $\mathrm{R}$ groups with distinct electronic properties. In general, the properties of these groups did not affect the reaction, and the 2-amino-1,3,4-thiadiazole pyrazole hybrids $4 \mathrm{a}-\mathrm{f}$ were obtained with yields from 50 to $56 \%$ (Figure 4).

Finally, all the structures of the synthesized compounds were elucidated based on ${ }^{1} \mathrm{H}$ and ${ }^{13} \mathrm{C}$ supplemented with $2 \mathrm{D}$ NMR measurements.

\section{Antiproliferative and Cytotoxic Activities}

All the novel trifluoromethylated pyrazoles derivatives $\mathbf{2 a}-\mathbf{f}, \mathbf{3} \mathbf{a}-$ f, and $4 \mathbf{a}-\mathbf{f}$ were evaluated against the promastigote form of $L$. amazonensis and the epimastigote form of T. cruzi. The results are reported as the concentration causing a 50\% inhibition in cell growth $\left(\mathrm{IC}_{50}\right)$. Additionally, the toxicity of compounds was evaluated against two different cell lines: epithelial cell $\mathrm{LLCMK}_{2}$ and macrophages J774A1. The antiproliferative and cytotoxic activities are shown in Table 2 , as well as the calculated selectivity index (SI). In general, 18 compounds showed antiproliferative activity against $L$. amazonensis and T. cruzi.

As shown in Table $\mathbf{2}$, the series of the compounds $\mathbf{2 a - f}$ exhibited $\mathrm{IC}_{50}$ values in the range 18.9 to $61.7 \mu \mathrm{M}$ against $L$. amazonensis. The unsubstituted phenyl ring led to the compound with the worst activity $\left(2 \mathrm{e}, \mathrm{IC}_{50}=61.7 \mu \mathrm{M}\right)$. Among the halogens, the para-bromo phenyl ring exhibited 


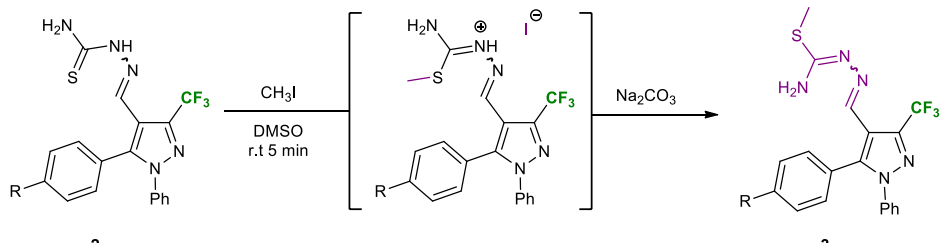

3<smiles>CSC(N)N/N=C/c1c(C(F)(F)F)nn(P)c1-c1ccc([N+](=O)[O-])cc1</smiles><smiles>CSC(N)N/N=C/c1c(C(F)(F)F)nn(-c2ccccc2)c1-c1ccc(F)cc1</smiles><smiles>CSC(N)N/N=C/c1c(C(F)(F)F)nn(-c2ccc(Cl)cc2)c1-c1ccccc1</smiles>

3a $(85 \%)$

3b $(78 \%)$<smiles>CSC(N)N/N=C/c1c(C(F)(F)F)nn(-c2ccccc2)c1-c1ccc(Br)cc1</smiles><smiles></smiles><smiles>COc1ccc(-c2c(C=NNC(N)SC)c(C(F)(F)F)nn2-c2ccccc2)cc1</smiles>

3d $(80 \%)$

3e $(73 \%)$

3f $(68 \%)$

FIGURE 3 | Substrate scope of thiosemicarbazones 3a-f. Reaction conditions: Thiosemicarbazone derivatives $\mathbf{2 a - f}\left(1.0 \mathrm{mmol}^{\prime}\right), \mathrm{Na}_{2} \mathrm{CO}_{3}(1.2 \mathrm{mmol})$, iodomethane $(1.0 \mathrm{mmol})$, DMSO $(5.0 \mathrm{ml})$. Isolated yield after recrystallization in hexane/ethyl acetate (4:1).

TABLE 1 | Optimization of the one-pot reaction to the synthesis of 4.

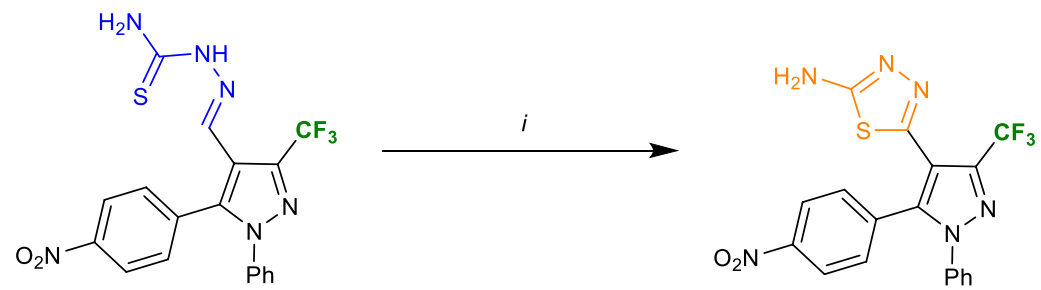

\begin{tabular}{|c|c|c|c|c|c|c|c|c|}
\hline \multirow{3}{*}{ Entry } & \multirow{3}{*}{ Solvent } & \multicolumn{2}{|c|}{$2 a$} & \multicolumn{4}{|c|}{$4 a$} & \multirow{3}{*}{ Yield $(\%)^{[\mathrm{b}]}$} \\
\hline & & $\mathrm{T}^{\circ} \mathrm{C}$ & \multirow{2}{*}{$\mathrm{Na}_{2} \mathrm{CO}_{3}$ (Eqv.) } & \multicolumn{2}{|c|}{ Oxidant } & \multirow[t]{2}{*}{ Time (hour) } & \multirow[t]{2}{*}{ Conversion $(\%)^{[\mathrm{a}]}$} & \\
\hline & & & & Oxidant & Eqv & & & \\
\hline 2 & THF & Reflux & 3.0 & $\mathrm{I}_{2}$ & 1.2 & 4 & 100 & 44 \\
\hline 3 & 1,4-dioxane & Reflux & 3.0 & $\mathrm{I}_{2}$ & 1.2 & 4 & 100 & 56 \\
\hline 4 & $\mathrm{EtOH}$ & Reflux & - & $\mathrm{FeCl}_{3}$ & 1.0 & 4 & 36 & - \\
\hline
\end{tabular}

${ }^{[a]}$ Conversion determined from the ${ }^{1} \mathrm{H}$ NMR spectrum of the crude product ${ }^{[b]}$ The product was dissolved in a mixture of hexane/ethyl acetate $(4: 1)$ and cooled to $0^{\circ} \mathrm{C}$, which induced crystallization.

the best activity $\left(\mathbf{2 d}, \mathrm{IC}_{50}=18.9 \mu \mathrm{M}\right)$, and was also the most active compound among the series $2 \mathbf{a}-\mathbf{f}$. The electronwithdrawing nitro group $\left(\mathbf{2 a}, \mathrm{IC}_{50}=26.7 \mu \mathrm{M}\right)$ and the electron-donating methoxy group $\left(2 \mathrm{f}, \mathrm{IC}_{50}=22.4 \mu \mathrm{M}\right)$ at the para position were also tolerated, presumably for steric reasons. In general, the compounds containing the bulkiest groups at the para position exhibited the best results against $L$. amazonensis. The selective index against macrophages ranged from 8 to 9 .

For the series of compounds $\mathbf{3 a}-\mathbf{f}$, again bulky groups such as bromo, methoxy, and nitro at the para position led to the most active compounds with $\mathrm{IC}_{50}$ values of $13.9,14.2$, and $18.3 \mu \mathrm{M}$, respectively. Furthermore, the methylation of the sulfur atom 


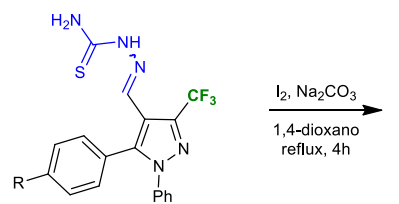<smiles>[R]c1ccc(-c2c(-c3nnc(N)s3)c(C(F)(F)F)nn2-c2ccccc2)cc1</smiles><smiles>Nc1nnc(-c2c(C(F)(F)F)nn(-c3ccccc3)c2-c2ccccc2)s1</smiles>

$4 a(56 \%)$<smiles></smiles>

4d $(54 \%)$<smiles></smiles>

$4 \mathrm{~b}(53 \%)$<smiles>Nc1nnc(-c2c(C(F)(F)F)nn(-c3ccccc3)c2-c2ccccc2)s1</smiles>

4e $(52 \%)$<smiles>Nc1nnc(-c2c(C(F)(F)F)nn(-c3ccccc3)c2-c2ccc(Cl)cc2)s1</smiles>

$4 c(54 \%)$<smiles>COc1ccc(-c2c(-c3nnc(N)s3)c(C(F)(F)F)nn2-c2ccccc2)cc1</smiles>

4f $(50 \%)$

FIGURE 4 | Substrate scope of thiosemicarbazones 4a-f. Reaction conditions: Thiosemicarbazone derivatives $\mathbf{2 a - f}(1.0 \mathrm{mmol}), \mathrm{Na}_{2} \mathrm{CO}_{3}(3.0 \mathrm{mmol})$, iodine (1.0 $\mathrm{mmol})$, dioxane $(5.0 \mathrm{ml})$. Isolated yield after recrystallization in hexane/ethyl acetate (4:1).

TABLE 2 | In vitro antiproliferative activity in L. amazonensis, T. cruzi and cytotoxicity in mammalian cells treated with the compounds.<smiles>NC(=S)N/N=C/c1c(C(F)(F)F)nn(-c2ccccc2)c1-c1ccc(Br)cc1</smiles>

2<smiles>CSC(N)=N/N=C/c1c(C(F)(F)F)nn(P)c1-c1ccc(Br)cc1</smiles>

3<smiles>Nc1nnc(-c2c(C(F)(F)F)nn(-c3ccccc3)c2-c2ccc(Br)cc2)s1</smiles><smiles>O=Cc1c(C(F)(F)F)nn(-c2ccc([N+](=O)[O-])cc2)c1-c1ccccc1</smiles>

5

\begin{tabular}{|c|c|c|c|c|c|c|c|c|c|}
\hline Comp. & $\mathbf{R}$ & L. amazonensis $\mid \mathrm{C}_{50}(\mu \mathrm{M})^{[a]}$ & T. cruzili ${ }_{50}(\mu \mathrm{M})^{[b]}$ & Epithelial cells $\mathrm{CC}_{50}(\mu \mathrm{M})^{[c]}$ & Macrophages $\mathrm{CC}_{50}(\mu \mathrm{M})^{[d]}$ & $\mathrm{SI}_{\mathrm{L}-\mathrm{E}}[\mathrm{e}]$ & $\mathrm{SI}_{\mathrm{L}-\mathrm{M}}^{[f]}$ & $\mathrm{SI}_{\mathrm{T}-\mathrm{E}}^{[g]}$ & $\mathrm{SI}_{\mathrm{T}-\mathrm{M}}^{[h]}$ \\
\hline $2 a$ & $\mathrm{NO}_{2}$ & $26.7 \pm 1.4$ & $45.7 \pm 5.0$ & $302.6 \pm 5.8$ & $243.1 \pm 3.6$ & 11.33 & 9.10 & 6.62 & 5.32 \\
\hline $2 b$ & F & $49.6 \pm 3.8$ & $69.3 \pm 2.9$ & $345.7 \pm 2.6$ & $202.7 \pm 5.9$ & 6.97 & 4.09 & 4.99 & 2.92 \\
\hline $2 c$ & $\mathrm{Cl}$ & $44.6 \pm 6.2$ & $49.2 \pm 3.1$ & $376.8 \pm 5.0$ & $402.2 \pm 8.3$ & 8.45 & 9.02 & 7.66 & 8.17 \\
\hline $2 d$ & $\mathrm{Br}$ & $18.9 \pm 1.1$ & $30.3 \pm 5.2$ & $148.3 \pm 6.9$ & $169.7 \pm 7.2$ & 7.85 & 8.98 & 4.89 & 5.60 \\
\hline $2 e$ & $\mathrm{H}$ & $61.7 \pm 2.9$ & $83.1 \pm 5.3$ & $394.4 \pm 7.1$ & $399.5 \pm 6.6$ & 6.39 & 6.47 & 4.75 & 4.81 \\
\hline $2 f$ & $\mathrm{MeO}$ & $22.4 \pm 3.0$ & $29.7 \pm 2.6$ & $154.8 \pm 2.6$ & $180.1 \pm 4.7$ & 6.91 & 8.04 & 5.21 & 6.06 \\
\hline $3 a$ & $\mathrm{NO}_{2}$ & $18.3 \pm 2.4$ & $24.1 \pm 0.8$ & $176.4 \pm 5.9$ & $221.4 \pm 3.1$ & 9.64 & 12.10 & 7.32 & 9.19 \\
\hline $3 b$ & $\mathrm{~F}$ & $34.3 \pm 4.7$ & $39.9 \pm 3.2$ & $119.4 \pm 3.0$ & $202.4 \pm 3.4$ & 3.48 & 5.90 & 2.99 & 5.07 \\
\hline $3 c$ & $\mathrm{Cl}$ & $30.7 \pm 2.2$ & $37.2 \pm 4.8$ & $297.1 \pm 6.1$ & $300.8 \pm 3.9$ & 9.68 & 9.80 & 7.99 & 8.09 \\
\hline $3 d$ & $\mathrm{Br}$ & $13.9 \pm 0.6$ & $25.7 \pm 2.5$ & $114.0 \pm 3.8$ & $146.7 \pm 3.6$ & 8.20 & 10.55 & 4.44 & 5.71 \\
\hline $3 e$ & $\mathrm{H}$ & $44.7 \pm 5.0$ & $62.4 \pm 3.8$ & $202.5 \pm 6.2$ & $326.4 \pm 4.5$ & 4.53 & 7.30 & 3.25 & 5.23 \\
\hline $3 f$ & $\mathrm{MeO}$ & $14.2 \pm 1.1$ & $19.1 \pm 0.9$ & $101.3 \pm 2.4$ & $122.9 \pm 3.1$ & 7.13 & 8.65 & 5.30 & 6.43 \\
\hline $4 a$ & $\mathrm{NO}_{2}$ & $19.6 \pm 1.5$ & $29.0 \pm 2.7$ & $211.3 \pm 3.1$ & $285.3 \pm 5.6$ & 10.78 & 14.56 & 7.29 & 9.84 \\
\hline $4 b$ & $\mathrm{~F}$ & $40.4 \pm 3.2$ & $41.6 \pm 5.3$ & $146.5 \pm 5.4$ & $249.3 \pm 6.2$ & 3.63 & 6.17 & 3.52 & 5.99 \\
\hline $4 c$ & $\mathrm{Cl}$ & $36.1 \pm 2.8$ & $40.5 \pm 3.1$ & $349.6 \pm 6.1$ & $385.2 \pm 5.8$ & 9.68 & 10.67 & 8.63 & 9.51 \\
\hline $4 d$ & $\mathrm{Br}$ & $14.7 \pm 0.9$ & $26.7 \pm 1.4$ & $120.1 \pm 2.5$ & $147.1 \pm 3.2$ & 8.17 & 10.01 & 4.50 & 5.51 \\
\hline $4 e$ & $\mathrm{H}$ & $52.4 \pm 3.5$ & $65.6 \pm 2.9$ & $264.3 \pm 3.2$ & $340.1 \pm 5.2$ & 5.04 & 6.49 & 4.03 & 5.18 \\
\hline $4 f$ & $\mathrm{MeO}$ & $17.1 \pm 1.6$ & $22.8 \pm 1.2$ & $133.9 \pm 5.1$ & $156.3 \pm 4.1$ & 7.83 & 9.14 & 5.87 & 6.86 \\
\hline 5 & $\mathrm{NO}_{2}$ & $73.1 \pm 2.8$ & $80.8 \pm 3.7$ & $421.6 \pm 12.3$ & $244.9 \pm 2.7$ & 5.76 & 3.35 & 5.22 & 3.03 \\
\hline
\end{tabular}

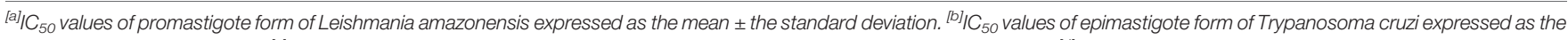

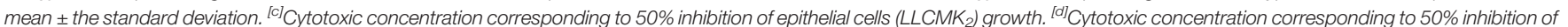

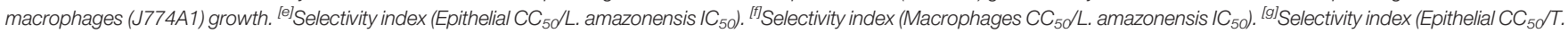
cruzi $\left.I C_{50}\right) .{ }^{[h]}$ Selectivity index (Macrophages $C_{50} / T$. cruzi $\left.I C_{50}\right)$. The best results are in bold. 
(3a-f) offered an improvement on activity, leading to compounds about 1.5 -fold more active compared to $\mathbf{2 a - f}$. The most active compound, 3d, exhibited a selectivity index of 10.55 against macrophages.

Among the series of the 2-amino-1,3,4-thiadiazole pyrazole hybrids $\mathbf{4 a - f}$, it was also found that the presence of bulky groups such as bromo, methoxy, and nitro at the para position led to the most active compounds with $\mathrm{IC}_{50}$ values of $14.7 \mu \mathrm{M}(\mathbf{4 d}), 17.1$ $\mu \mathrm{M}$ (4f), and $19.6 \mu \mathrm{M}(\mathbf{4 a})$, respectively. These compounds showed SI in the range 9.14 to 14.56 . Moreover, the molecular hybridization of 2-amino-1,3,4-thiadiazole and pyrazole moieties offered the most potent compounds (4a-f) compared to their thiosemicarbazone analogues (2a-f), demonstrating the importance of the heterocyclic ring.

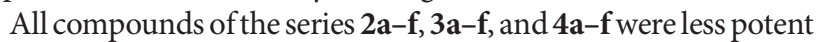
against $T$. cruzi compared to $L$. amazonensis. The most active compounds for L. amazonensis (2d, 3d, and 4d) were about 2fold less potent for T. cruzi. Again, the compounds with bulky groups at the para position had the best $\mathrm{IC}_{50}$ values in each series.

Aiming to evaluate the influence of the thiosemicarbazone group attached at the 3-position of the pyrazole core (2a), we choose to assess the antileishmanial and antitrypanosomal activities of the 4-formyl pyrazole derivative 5a. The insertion of the formyl group at the 4-position of the pyrazole ring led to a 3 -fold and 2-fold decrease in activity against L. amazonensis and T. cruzi, respectively. Compound 5a also exhibited a worse SI compared to $2 \mathrm{a}$.

It is worth mentioning that comparing the values $\mathrm{IC}_{50}$ of the series $\mathbf{2 a - f}$ with $\mathbf{3 a}-\mathbf{f}$ and $\mathbf{4 a - f}$ it is observed a low variance between the results obtained. In general, the compounds of the series $3 \mathbf{a}-\mathbf{f}$ were 1.4 -fold most active compared to $\mathbf{2} \mathbf{a}-\mathbf{f}$, and the compounds $4 \mathbf{a}-\mathbf{f}$ were 1.2 -fold most active than $2 \mathbf{a}-\mathbf{f}$. Comparing the works of Batista (Batista et al., 2019) and Vandresen (Vandresen et al., 2017), the transformation of the thiosemicarbazone group into 2-amino-1,3,4-thiadiazole derivative led to compounds in the range 1.4 to 6 -fold less actives.

\section{CONCLUSIONS}

In summary, we synthesized 18 new trifluormethylated pyrazole hybrids by a simple and efficient methodology. Screening of the compounds against Leishmania amazonensis and Trypanosoma cruzi demonstrated the importance of the substitution pattern of the trifluoromethylated $N$-aryl pyrazole system. The presence of a bulky group such as bromo, methoxy, or nitro at the para position of the aryl ring attached at the 5-position of pyrazole was essential for antiparasitic activity. Also, the nature of the substituent attached at the 3-position of pyrazole influenced the

\section{REFERENCES}

Alvar, J., Vélez, I. D., Bern, C., Herrero, M., Desjeux, P., Cano, J., et al. (2012). Leishmaniasis worldwide and global estimates of its incidence. PLoS One 7 (5), e-35671. doi: 10.1371/journal.pone.0035671 activity, as the thiosemicarbazone derivative led to active compounds, whereas the formyl derivative was inactive. Furthermore, the transformation of the thiosemicarbazone into $S$-methyl and 2-amino-1,3,4-thiadiazole derivatives increased the activity against both protozoans. However, the compounds were more active against L. amazonensis. From the results obtained in this study, the $S$-methyl thiosemicarbazones $\mathbf{3 a}, \mathbf{3 d}$, and $\mathbf{3} \mathbf{f}$, and 2-amino-1,3,4-thiadiazole pyrazole hybrids $\mathbf{4 a}, \mathbf{4 d}$, and $\mathbf{4 f}$ could be considered as lead structures for the optimization of antileishmanial and antitrypanosomal properties.

\section{DATA AVAILABILITY STATEMENT}

All datasets presented in this study are included in the article/ Supplementary Material.

\section{AUTHOR CONTRIBUTIONS}

JC and KP contributed to the synthesis, analysis of the results, and writing of the paper. MS contributed to synthesis and formal analysis. DL-B and HV contributed to pharmacological analyses. SM contributed to the HRMS analysis. CN contributed to the supervision of pharmacological analyses. FR contributed to designing the work, synthesis supervision, project administration, funding acquisition, and in the review and editing of the writing. All authors contributed to the article and approved the submitted version.

\section{FUNDING}

Coordenação de Aperfeiçoamento de Pessoal de Nível SuperiorCAPES/Brazil (AUXPE-PROEX-CAPES-Processo $n^{\circ}$ 23038.000872/2018-83).

\section{ACKNOWLEDGMENTS}

The authors are grateful for the financial support and fellowships from CAPES/Brazil.

\section{SUPPLEMENTARY MATERIAL}

The Supplementary Material for this article can be found online at: https://www.frontiersin.org/articles/10.3389/fphar.2020.591570/ full\#supplementary-material

Batista, S. A. A., Vandresen, F., Falzirolli, H., Britta, E., Oliveira, D. N., Catharino, R. R., et al. (2019). Synthesis and comparison of antileishmanial and cytotoxic activities of $S$-(-)-limonene benzaldehyde thiosemicarbazones with their $R-(+)$-analogues. J. Mol. Struct. 1179, 252-262. doi: 10.1016/j.molstruc.2018.11.017

Bekhit, A. A., Hassan, A. M., Abd El Razik, H. A., El-Miligy, M. M., Elagroudy, E. J., and Bekhit, A.-D. (2015). New heterocyclic hybrids of pyrazole and its bioisosteres: 
Design, synthesis and biological evaluation as dual acting antimalarial antileishmanial agents. Eur. J. Med. Chem. 94, 30-44. doi: 10.1016/ j.ejmech.2015.02.038

Chain, C. Y., Couto, D. E. P., Sbaraglini, M. L., Labriola, C. A., Millone, M. A. D., Ramirez, E. A., et al. (2019). Trypanosoma cruzi virulence factors for the diagnosis of Chagas' disease. ACS Infect. Dis. 5 (11), 1813-1819. doi: 10.1021/ acsinfecdis.9b00269

De Melos, J. L. R., Torres-Santos, E. C., Faiões, V., Dos, S., De Nigris Del Cistia, C., Sant'anna, C. M. R., et al. (2015). Novel 3,4-methylenedioxyde-6-Xbenzaldehyde-thiosemicarbazones: Synthesis and antileishmanial effects against Leishmania amazonensis. Eur. J. Med. Chem. 103, 409-417. doi: 10.1016/j.ejmech.2015.09.009

Freitas, R. H. C. N., Barbosa, J. M. C., Bernardino, P., Sueth-Santiago, V., Wardell, S. M. S. V., Wardell, J. L., et al. (2020). Synthesis and trypanocidal activity of novel pyridinyl-1,3,4-thiadiazole derivatives. Biomed. Pharmacother. 127:110162. doi: 10.1016/j.biopha.2020.110162

Goupil, L. S., and McKerrow, J. H. (2014). Introduction: Drug Discovery and Development for Neglected Diseases. Chem. Rev. 114, 11131-11113. doi: $10.1021 / \mathrm{cr} 500546 \mathrm{~h}$

Gradoni, L., López-Velez, R., and Mokni, M. (2017). Manual on case management and surveillance of the leishmaniases in the WHO European Region (Copenhage: WHO Regional Office for Europe). Available at: https://www. euro.who.int/en/publications/abstracts/manual-on-case-management-andsurveillance-of-the-leishmaniases-in-the-who-european-region-2017.

Habercom, G. E. (2016). Weekly epidemiological record. WHO 91 (22), 285-296. https://www.who.int/wer/2016/wer9122/en/.

Haider, S., Alam, M. S., and Hamid, H. (2015). 1,3,4-thiadiazoles: A potent multi targeted pharmacological scaffold. Eur. J. Med. Chem. 92, 156-177. doi: 10.1016/j.ejmech.2014.12.035

Hu, Y., Li, C. Y., Wang, X. M., Yang, Y. H., and Zhu, H. L. (2014). 1,3,4thiadiazole: synthesis, reactions, and applications in medicinal, agricultural, and materials chemistry. Chem. Rev. 114, 5572-5610. doi: 10.1021/cr400131u

Hurrell, B. P., Regli, I. B., and Tacchini-Cottier, F. (2016). Different leishmania species drive distinct neutrophil functions. Trends Parasitol. 32 (5), 392-401. doi: $10.1016 /$ j.pt.2016.02.003

Jain, A. K., Sharma, S., Vaidya, A., Ravichandran, V., and Agrawa, R. K. (2013). 1,3,4-thiadiazole and its derivatives: A review on recent progress in biological activities. Chem. Biol. Drug Des. 815, 557-576. doi: 10.1111/cbdd.12125

Martins, S. C., Desoti, V. C., Lazarin-Bidóia, D., Vandresen, F., Da Silva, C., UedaNakamura, T., et al. (2016). Synthesis and evaluation of the trypanocidal activity of a series of 1,3,4-thiadiazoles derivatives of R-(+)-limonene benzaldehydethiosemicarbazones. Med. Chem. Res. 25, 1193-1203. doi: 10.1007/s00044-016-1561-7

Monteiro, M. E., Lechuga, G., Lara, L. S., Souto, B. A., Viganó, M. G., Bourguignon, S. C., et al. (2019). Synthesis, structure-activity relationship and trypanocidal activity of pyrazole-imidazoline and new pyrazole-tetrahydropyrimidine hybrids as promising chemotherapeutic agents for Chagas disease. Eur. J. Med. Chem. 182:111610. doi: 10.1016/j.ejmech.2019.111610

Petrov, V. A. (2009). Fluorinated Heterocyclic Compounds: Synthesis, Chemistry, and Applications (New Jersey: Wiley).

Pianoski, K. E., Poletto, J., Da Silva, M. J. V., Camargo, J. N. A., Jacomini, A. P., Gonçalves, D. S., et al. (2020). 1,2-Addition to trifluoromethylated $\beta$-enamino diketones: Regioselective synthesis of trifluoromethyl-containing azomethine pyrazoles and isoxazoles. Org. Biomol. Chem. 18, 2524-2537. doi: 10.1039/ d0ob00319k

Sangshetti, J. N., Khan, F. A. K., Kulkarni, A. A., Arote, R., and Patil, R. H. (2015). Antileishmanial drug discovery: Comprehensive review of the last 10 years. RSC Adv. 5, 32376-32415. doi: 10.1039/C5RA02669E

Serban, G. (2019). Future prospects in the treatment of parasitic diseases: 2amino-1,3,4-thiadiazoles in leishmaniasis. Molecules 24 (8):1557. doi: 10.3390/ molecules 24081557

Vandresen, F., Souza, M. R. P., Britta, E., Silva, E. L., Carvalho, J. E., Ruiz, A. L. T. G., et al. (2017). Avaliação das atividades antiproliferativa e antileishmania de 2-amino-5-aril-1,3,4-tiadiazóis derivados do $R$ (+)-limoneno. Rev. Virtual Quim. 9 (3), 1285-1302. doi: 10.21577/19846835.20170075

World Health Organisation (2020). Neglected tropical diseases. Available at: http:// www.who.int/neglected_diseases/diseases/en/ (Accessed July 15, 2020).

Conflict of Interest: The authors declare that the research was conducted in the absence of any commercial or financial relationships that could be construed as a potential conflict of interest.

Copyright (c) 2020 Camargo, Pianoski, dos Santos, Lazarin-Bidóia, Volpato, Moura, Nakamura and Rosa. This is an open-access article distributed under the terms of the Creative Commons Attribution License (CC BY). The use, distribution or reproduction in other forums is permitted, provided the original author(s) and the copyright owner(s) are credited and that the original publication in this journal is cited, in accordance with accepted academic practice. No use, distribution or reproduction is permitted which does not comply with these terms. 